\title{
Available connectivity analysis under free flow state in VANETs
}

\author{
Chen Chen", Lei Liu, Xiaobo Du, Xiaolu Wei and Changxing Pei
}

\begin{abstract}
Emerging of inter-vehicle communication gives vehicles opportunities to exchange information within limited radio ranges and self-organize in Ad Hoc manner into Vehicular Ad Hoc Networks (VANETs). However, due to strong mobility, limited market penetration rate, and lack of roadside units, connectivity is obviously a scarce resource in VANETs. Further, only depending on direct connectivity, i.e. one-hop connected links between vehicles, is far from the continuous growing communication demands in VANETs, such as inter-vehicle amusement, cooperative collision avoidance, inter-vehicle emergency notification etc. Therefore, the indirect connectivity from multi-hop forwarding and store-carry-forward strategy is also a necessary and powerful complement especially to the case where direct connections are hardly obtained. In this article, we define a new metric named available connectivity which involves both direct and indirect connectivity. By deep analyzing the statistical properties of direct and indirect connectivity in free flow state, the proposed available connectivity is obtained and quantified to increase the information dissemination opportunities for vehicles especially in a relatively slow topology changing scenario. Numerical results show that the available connectivity could provide better references for different VANETs applications and has potential relationships with many network parameters.
\end{abstract}

Keywords: Vehicular Ad Hoc Networks, Connectivity, Mobility

\section{Introduction}

Vehicular Ad hoc Networks [1] (VANETs) are distributed, self-organizing communication networks built up by moving vehicles, and are thus characterized by very strong node mobility and limited degrees of freedom in the mobility patterns. The discussed IEEE 1609 Wireless Access in Vehicular Environments [2] draft is being developed for VANETs applications including mainly safety-related scenarios, such as Cooperative forward Collision Warning [3] (CCW) system, traffic signal violation warning [4], lane change warning [5] and some information applications [6]. All these applications greatly rely on the successful packets exchanging which is based on reliable links or node connectivity on a road segment. Thereupon, network connectivity is fundamental and crucial to any practical application in VANETs. Due to mobility, vehicles on a road segment can not only communicate with each other directly, they can also deliver the packets indirectly to the receiver by store-carry or

\footnotetext{
* Correspondence: cc2000@mail.xidian.edu.cn

State Key Laboratory of Integrated Services Networks, Xidian University, Xian 710071, China
}

multi-hop forwarding. Although connectivity can be obtained directly or indirectly, it is still a limited resource especially during sparse communication environment such as on highway or with low market penetration ratio [7], i.e. the number of equipped vehicles to the number of total vehicles. Limited connectivity directly affects the possible speed and range at which information can be disseminated over a VANET, and hence limits the up-todatedness of the shared information that can be achieved.

In summary, connectivity in VANET is important for us to analyze and evaluate the overall network performance. Many schemes, such as stochastic process [8], Gaussian unitary ensemble of random-matrix theory [9] queuing theory [10] etc., have been proposed to calculate the probability of connectivity with different premises in VANETs. Their works all discussed connectivity based on the famous 3-state traffic theory in which the fundamental relation of traffic parameters on a road is given by the following equation:

$$
F=S \times K,
$$

where $\mathrm{F}, \mathrm{S}$, and $\mathrm{K}$ are the traffic flow, average speed, and traffic density, respectively. The influence of above three 
parameters on connectivity can be disclosed from Figure 1. Before the state transforming point, which indicates the position where packets begin to be queued in vehicles' sending buffers with vehicles density increasing, the connectivity may be intermittent and is worth of studying during the so-called free flow state expressed by solid line. However, after the state transforming point, which is depicted by dashed line as the congestion flow state, the connectivity is guaranteed by larger density and the road is often jammed by vehicles with closing inter-vehicle distances within which direct connectivity can be readily obtained. Therefore, in this state, the connectivity is not still important and schemes for congestion control will rise to the first priority. The state denoted by the solid line is defined as the free flow state and we certainly discuss the available connectivity in this state throughout our article.

The rest of the article is organized as follows. The following section provides the motivation behind our works and discusses the possible usages of available connectivity in VANETs. Section "Related studies" presents the related works. Section "Available connectivity definition and assumption" introduces our definition of available connectivity and assumptions. In Section "Statistical analysis of available connectivity", the statistical analysis of direct and indirect connectivity is proposed. Numerical results and evaluation are presented in Section "Numerical results". Finally, this article ended with some conclusion given in the last section.

\section{Motivation}

Connectivity in Ad Hoc networks has a mature body of research, but it has been less studied in VANETs. However, With VANETs stepping into our daily life in a big step, connectivity has played an important role in many related applications to ensure our driving safety and increase our comfortableness. For instance, in CCW scenario, a well connectivity could help to avoid the

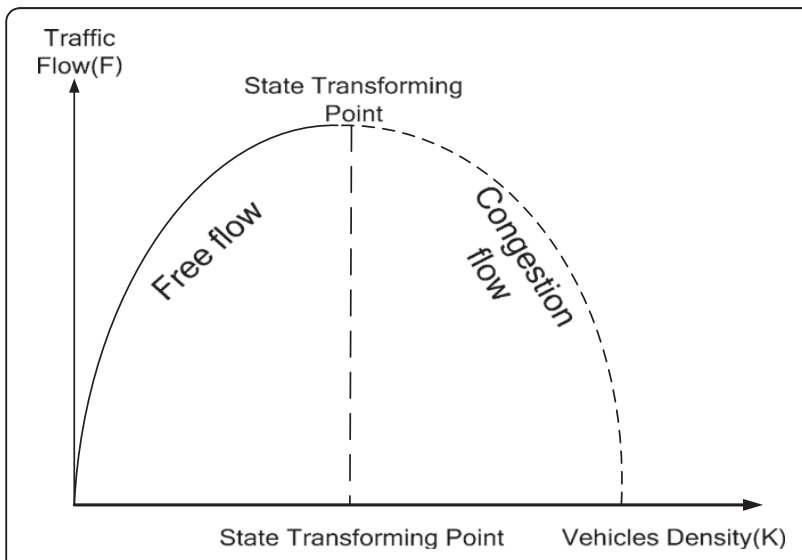

Figure 1 Relation between basic parameters in vehicle traffic. series collisions by disseminating the warning messages farther and quicker. In Figure 2, the red car crashed with the blue one and sent a CCW message. Car 1 could receive the $\mathrm{CCW}$ by direct connection and may brake in time to avoid crash. But, without indirect connection, i.e, multi-hop forwarding here, cars 2 and 3 will inevitably crash if the distances between them and the front cars are within unsafe range [11]. In Figure 3, vehicles 2-5 could detect the front traffic jam by the messages storecarry-forwarded by vehicle 1 from the red sender. Therefore, owing to indirect connectivity, they can choose to enter into auxiliary road to avoid traffic jam and save time. In summary, connectivity, direct or indirect, could provide more opportunities to vehicles to decide their action wisely and safely and to achieve better experiences. Hence, a good definition of connectivity or metric to measure connectivity directly or indirectly is necessary in VANETs.

In this study, we define the connectivity as the number of achievable connections directly or indirectly. The definition can explore the maximum possibility for messages to be widely or quickly transmitted especially in safety-related applications contexts. The connection possibility is investigated on statistical characteristics in terms of either connected duration length or inter-vehicle distances. To reflect the high dynamism of connectivity due to different mobility in VANETs, the influence of velocity is also introduced.

The elaborate investigation to available connectivity in VANETs can make many otherwise complex problems easy. For example, to design an admission control strategy in VANETs, available connectivity can provide important reference for connectivity improvement by introducing specific vehicles. To evaluate the performance of emergent applications in VANETs, available connectivity can directly determine the successful delivery ratio of emergency notifications through given vehicles. On the other hand, to spread out the emergency notification messages quick and far enough, available connectivity could also be taken as a better choosing criterion or metric for effective forwarders. To route the packets based on available connectivity, throughput improvement can readily be obtained and backup multi-path may also be figured out by different links. In a dense network, available connectivity can also offer a threshold reference on packet generation rate to maintain sufficient connectivity but does not make the overall network congested. In summary, based on the full analysis to the proposed available connectivity, many works could be done to improve the safety, comfortableness and efficiency in VANETs.

\section{Related studies}

Although connectivity analysis is a classical problem in wireless communication networks, it has been a hot topic of interests in VANETs, especially due to the 


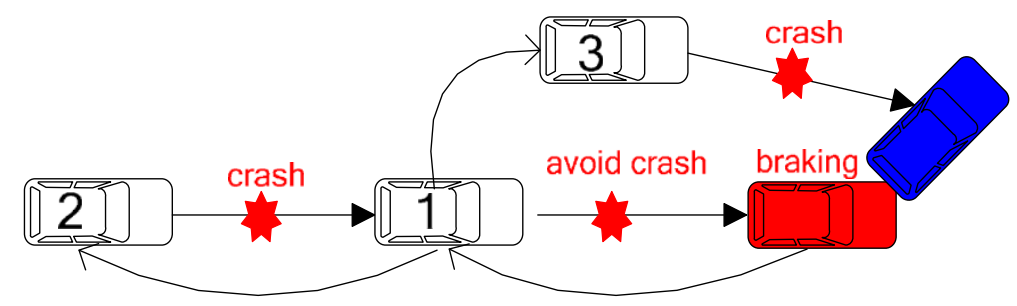

Figure 2 CCW application scenario.

recently increasing research activities. From our viewpoint, the connectivity research in VANETs can be classified into two categories: one for investigating the lifetime properties of connected links or paths; another for investigating the inter-vehicle distances.

Researches on lifetime properties of connectivity focus on the statistical analysis of connection duration length. The authors of [8] discussed the connection duration length expression in detail considering the velocity vector involving movement directions. The numerical results in their article found high relative velocities imposing a hard task on some cooperative maneuvers including underlying routing protocols. In their work, the short connection duration was enough for emergency notification scenarios. In [12], the connection duration length between two vehicles was figured out, given their speed, direction and radio ranges. According to the obtained connected period equation, an admission control strategy was proposed to determine whether the next vehicle is allowed to inject to the current traffic flow without disrupting the ongoing connections. In [13], metrics for evaluating node connectivity in VANETs had been proposed considering the different nodal mobility effects. The connection duration length distribution was characterized by average duration of the k-hop path existing between any two nodes or vehicles. They also showed by simulation that multi-hop paths have much poorer connectivity statistics than single-hop ones in VANETs.
Researches on the connectivity statistics of inter-vehicle distances focus on disclosing the relationship between connectivity and connection distance which is defined as the length of the connected path. In [8], vehicular distance distributions had been expressed by a tuple $(h, v, p)$, where $h$ denotes the inter-vehicle distance, $v$ for velocity and $p$ as market penetration ratio, respectively. They derived the connectivity formula in terms of distance between two nodes given specific propagation model and radio range. The expression was also further extended to the connectivity of a node with degree $n$. In [10], the authors introduced an equivalent $M / D / \infty$ queuing model to obtain the Laplace transform of the tail probability of the connection distance and an explicit form for the expected value of the connection distance. Their numerical results mainly showed that increasing the traffic flow and the vehicles' radio range led to the increasing of the metrics of connectivity in term of platoon size and connectivity distance. The authors of [14] showed that, under free flow state, connectivity increases as either the vehicular density or the number of lanes growing. Especially, the distances had a major impact on connectivity when it is $3-4$ times farther than the radio range. Beyond this distance, the connectivity declines slowly. In [15], a simple geometric analysis of connectivity was proposed in vehicular networks under a distance-based radio communication model. They presented the notion of connectivity robustness based on inter-vehicle distances, which can generate a locally computable function providing a sufficient

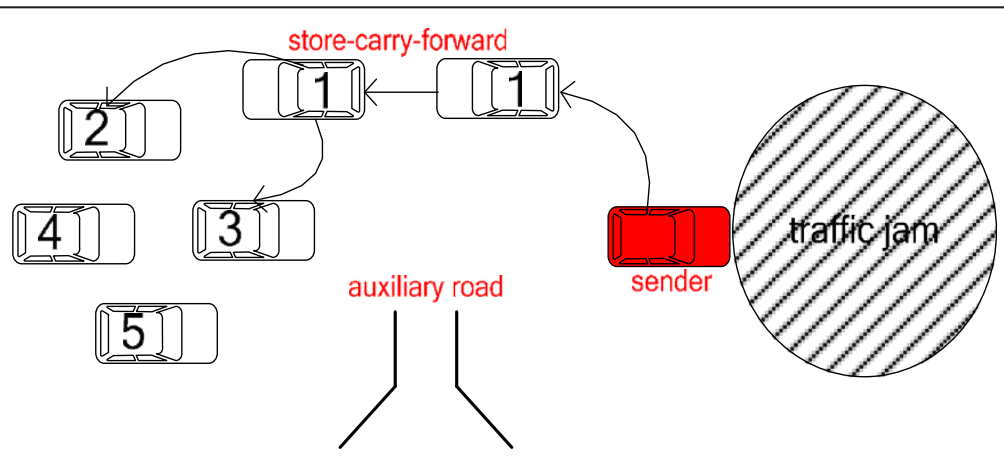

Figure 3 Traffic jam avoidance. 
reference for connectedness of the network. With the aforementioned researches, although, there were also lots of works deducing or expressing connectivity by vehicles density [16,17], speed or equivalent speed [18], transmission range $[16,19]$ etc., they all can be further transformed to the equivalent expression of statistical distributions of connection duration length or inter-vehicle distances. Further, generally speaking, it is difficult to give a uniform or general Probability Density Function (PDF) or Cumulative Distribution Function (CDF) of the inter-vehicle distances or connection duration length under different application scenarios such as overtaking, platoon and collisions. However, when the traffics are in the free-flow state, some distribution function and the probability of minimum node degree could be calculated. Therefore, we focus on the connectivity statistical analysis in free flow state.

\section{Available connectivity definition and assumption}

To fully utilize the connected direct or indirect links, an elaborate statistical analysis to the available connectivity should be given first for disclosing the influential factors. The definition of available connectivity in our study is given as follows:

Definition 1: Available connectivity for a vehicle in VANET is defined as the number of achievable connected links from it within a given period.

Available connectivity, including direct and indirect connectivity, can meet the continuously growing communication demands on road. Direct connectivity means one-hop connected links between nodes, namely, these nodes could communicate with each other directly, and there does not need relay nodes as shown in Figure 4. Indirect connectivity consists of multi-hop forwarding and store-carry-forward. Nodes corresponding to indirect connectivity could not communicate directly with each other and introduction of relay nodes or forwarders is necessary, as shown in Figure 5.

In VANETs, due to the high mobility of vehicles, topology is time-variant and the achievable connected

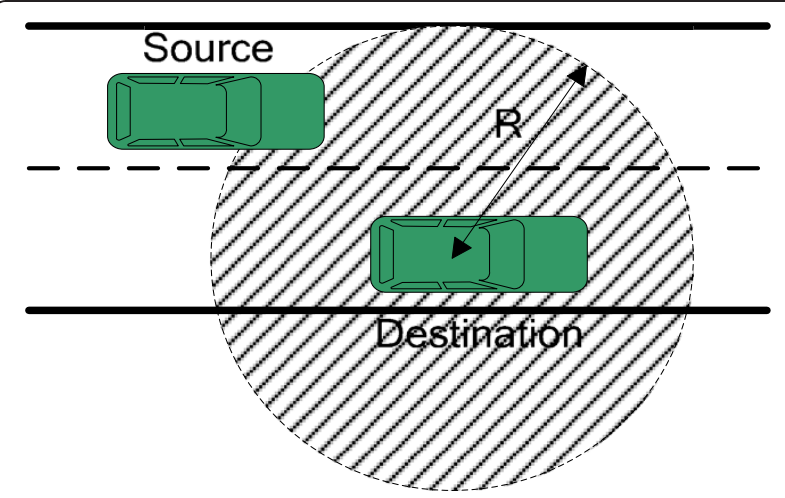

Figure 4 Reference setup for direct connectivity. links for a given vehicle are also changing frequently. Therefore, accurate definition of available connectivity should reflect the instantaneous state. However, although instantaneous connectivity is precise, it is no use for us to introduce such momentary parameter or reference for future design. As a result, we intend to define available connectivity within a given period. Correspondingly, the discussed scenario in this study just considers the slow changing topology case which is accurately reasonable especially under free flow state.

Let $\delta$ be the investigation period, i.e. we calculate available connectivity of a given node every $\delta$ seconds.

Theorem 1: The available connectivity of any given vehicle $i$ at time $\mathrm{T}$ is defined as:

$$
A C(T)=N_{i}(T)+\sum_{j} \theta_{i}(j, T)
$$

where $T \in\{0, \delta, 2 \delta, \ldots\}$.

$A C(T)$ denotes the available connectivity during $[T-\delta, T]$, and $N_{i}(T)$ is the number of neighbors around $i$ at time T. $\theta_{i}(j, T)$ is a probability of the achievable indirect connections at time $\mathrm{T}$.

$A C(T)$ represents the number of achievable links that the reference node, i.e. the investigated node for available connectivity analysis, could obtain during $[T-\delta, T]$. High available connectivity means there are more potential nodes which can connect with the reference node. According to available connectivity, we can control speeds of vehicles and the radio range to realize high connectivity in a VANET.

\section{Statistical analysis of available connectivity}

In this section, the available connectivity will be analyzed in detail by inspecting the connection properties in direct and indirect scenarios, respectively. Thus, the final available connectivity can be obtained by combining the obtained analysis results. For explanation simplicity, we use different analyzing method for connectivity study in different scenarios as follows.

\section{Direct connectivity}

In this section, we will investigate the direct connectivity for a given reference node in VANETs. The result will be given in terms of PDF of the connection duration length in free flow state.

For a reference node, the one-hop neighbors or the nodes within the radio range of the reference node, contribute to the direct connectivity. Without loss of generality, we just investigate the contribution to direct connectivity from one specific neighbor in later discussion. Results for others are similar and will be combined when final available connectivity is calculated. 


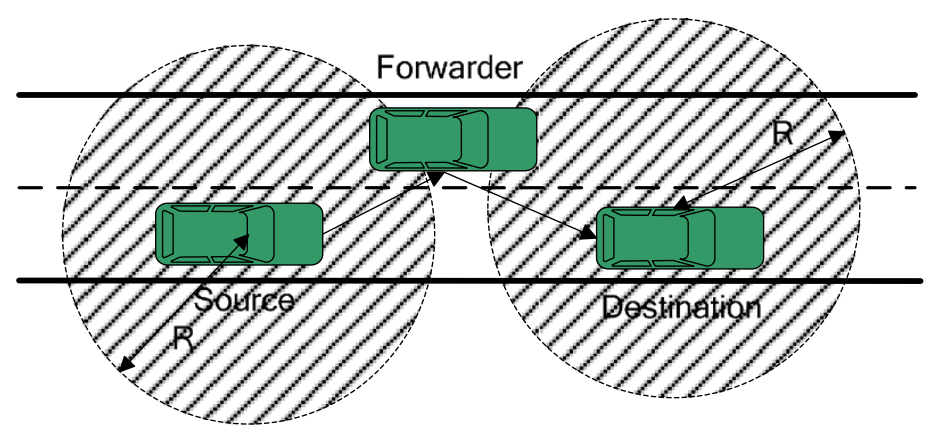

(a) Multi-hop forwarding strategy

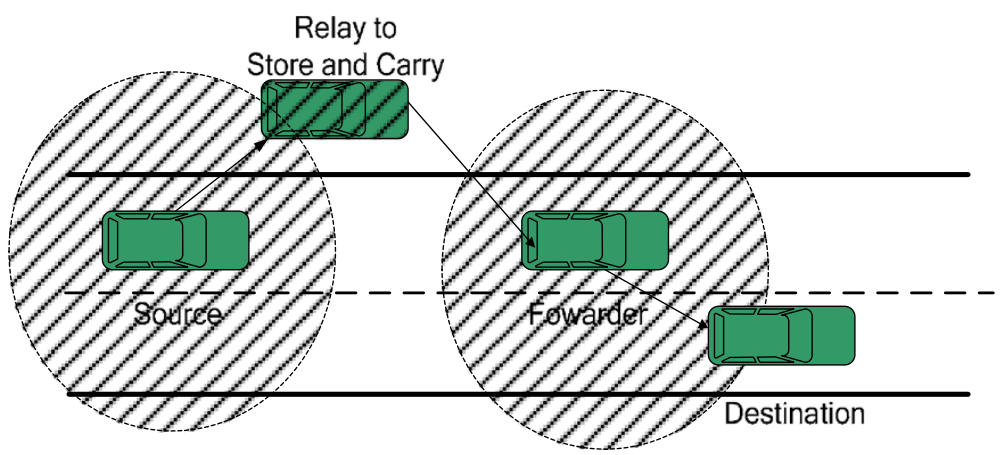

(b) Store-carry-forward strategy

Figure 5 Reference setup for indirect connectivity.

We assume $i$ and $j$ are two vehicles in a VANET driving in the same or opposite direction as shown in Figure 6. $d_{i, j}$ is the Euclidian distance between them. Let $i$ be the reference node.

Theorem 2: The duration of connection between $i$ and $j$ is

$$
\tau=\frac{2}{|v|^{2}} \sqrt{|v|^{2} r^{2}-\left(p_{y} v_{x}-p_{x} v_{y}\right)^{2}}
$$

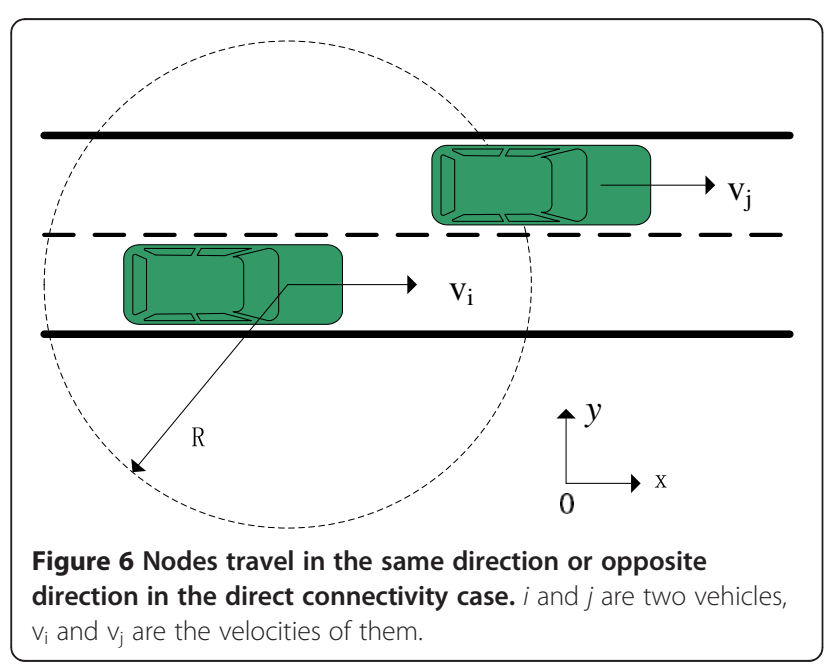

where $v=\left(v_{x}, v_{y}\right)^{T}$ is the relative speed vector and $p=\left(p_{x}, p_{y}\right)^{T}$ is the relative position vector. $r$ is the communication radio range.

Proof If the radio range $r$ is greater than $d_{i, j}$, then $i$ and $j$ can be named as a connected node pair. In other words, vehicles $i, j$ are connected and could communicate with each other directly via a radio channel. Let $p_{i, j}$ and $v_{i, j}$ be the position and speed vectors of vehicle $i, j$ respectively. If $i$ and $j$ are within each other's radio range at time $t$, we obtain the following inequality:

$$
\left\|\left(p_{i}+v_{i} \cdot t\right)-\left(p_{j}+v_{j} \cdot t\right)\right\| \leq r
$$

With $p=p_{i}-p_{j}=\left(p_{x}, p_{y}\right)^{T}, v=v_{i}-v_{j}=\left(v_{x}, v_{y}\right)^{T}$, we get

$$
\|p+v \cdot t\| \leq r
$$

If possible, the above inequality results in two solutions, i.e. $t_{1}$ and $t_{2}$, where $t_{1}$ and $t_{1}$ are denoted as the occasions when nodes $i$ and $j$ enter and leave radio ranges of each other respectively. Thus, we can 
obtain the duration of connection between $i$ and $j$ as $\mathrm{\tau}=\left|t_{1}-t_{2}\right|$, where

$$
\begin{aligned}
\tau= & \frac{2}{v_{y}^{2}+v_{y}^{2}} \sqrt{\left(v_{x}^{2}+v_{y}^{2}\right) r^{2}+2 p_{x} p_{y} v_{x} v_{y}-p_{y}^{2} v_{x}^{2}} \\
& -p_{x}^{2} v_{y}^{2} \\
= & \frac{2}{|v|^{2}} \sqrt{|v|^{2} r^{2}-\left(p_{y} v_{x}-p_{x} v_{y}\right)^{2}}
\end{aligned}
$$

By Equation (6), it can be noticed that vehicles in congestion flow state are almost always connected and this case could last long time (compared to vehicles in free-flow state) because of their small velocity differences. In other words, a deep analysis to direct connectivity in congestion flow state makes no sense. Hence, we focus on the direct connectivity in free flow state. The authors of [18] proved that the speeds of vehicles in free flow state follow a normal distribution. To confirm this result, we also cite the real measured data on Peking Second Ring Road. Table 1 shows the collected cumulative occurrence frequency for given velocity versus inter-vehicle distances and corresponding fitted normal distribution parameters, where $\mathrm{h}$ indicates the inter-vehicle distance and $\mathrm{v}$ denotes the speed.

With above conclusion, we can give the $\operatorname{PDF} f_{V}\left(v_{i}\right)$ for velocity $v_{i}$ of vehicle $i$ as:

$$
f_{V}\left(v_{i}\right)=\frac{1}{\sqrt{2 \pi} \sigma_{i}} e^{-\frac{\left(v_{i}-\mu_{i}\right)^{2}}{2 \sigma_{i}^{2}}}
$$

where $u_{i}$ and $\sigma_{i}$ are the average velocity and the standard deviation respectively. The distances of successive vehicles in a highway in free flow state are generally not too near because the traffic flow is free or light-load and overtaking is allowed. Thereupon, the speeds of vehicles

\begin{tabular}{|c|c|c|c|c|c|c|}
\hline $\mathbf{v}$ & $<8$ & 8-18 & $18-28$ & $28-38$ & $38-48$ & $>48$ \\
\hline \multicolumn{7}{|l|}{$\mathrm{h}$} \\
\hline$<15$ & 22 & 95 & 105 & 36 & 1 & 0 \\
\hline $15-20$ & 0 & 21 & 66 & 30 & 9 & 0 \\
\hline 20-30 & 0 & 28 & 84 & 51 & 17 & 0 \\
\hline $30-50$ & 0 & 0 & 22 & 34 & 13 & 4 \\
\hline$>50$ & 0 & 0 & 14 & 29 & 31 & 20 \\
\hline \multicolumn{3}{|c|}{ Samples $=732$} & \multicolumn{2}{|c|}{$\mu=40.15$} & \multicolumn{2}{|c|}{$\sigma=11.744$} \\
\hline
\end{tabular}
are unrelated or in other words, all vehicles' velocities here are Independent Identically Distributed (I.I.D) stochastic variables [20]. Hence, the velocity difference $v_{d}$ of two nodes $i$ and $j$ is distributed according to the PDF as follows:

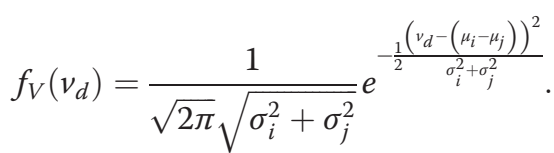

The mean velocity difference is $\mu=\mu_{i}-\mu_{j}$ and the standard deviation of velocity difference is $\sigma=\sqrt{\sigma_{i}^{2}+\sigma_{j}^{2}}$.

In practice, each vehicle's velocity may actually not be much bigger or very close to zero in the free flow traffic state. Therefore, the speed bounds $v_{\min }$ and $v_{\max }$, denoting the lower and upper limit of velocity respectively, are introduced, where $v_{\min }$ and $v_{\max }$ are further defined as $v_{\min }=\mu-3 \sigma, v_{\max }=\mu+3 \sigma$. The reason behind these two speed limits is that the area in range the $(\mu-3 \sigma, \mu+3 \sigma)$ covers about $99.7 \%$ of the whole area for a normal distribution curve. Accordingly, we revise the PDF of velocity with speed limits as:

$$
f_{T V}(v)=\frac{f_{V}(v)}{\int_{v_{\min }}^{v_{\max }} f_{V}(x) d x},
$$

where $v_{\min } \leq v \leq v_{\max }$.

The aforementioned discussions consider that vehicles all drive in a line. However, the result is different when vehicles running on neighboring lanes.

Theorem 3: In free flow state, the PDF for connection duration length when vehicles run on neighboring lanes is

$$
f_{D C}(\tau)=\frac{s}{\tau^{2}} \frac{f_{v}\left(\frac{s}{\tau}\right)+f_{v}\left(-\frac{s}{\tau}\right)}{Q\left(\frac{v_{\min }-\mu}{\sigma}\right)-Q\left(\frac{v_{\max }-\mu}{\sigma}\right)} \tau \geq 0
$$

where $Q(x)=\frac{1}{2} \operatorname{erfc}\left(\frac{x}{\sqrt{2}}\right)$, and $\operatorname{erfc}(\cdot)$ is the complementary error function.

Proof In the case that vehicles run on neighboring lanes, i.e. $\mathrm{i}$ and $\mathrm{j}$ move paralleling, we could get $p=\left(p_{x}, p_{y}\right)^{T}$, $v=\left(v_{i}-v_{j}, 0\right)^{T}$, where $p_{x}^{2}+p_{y}^{2} \leq R^{2}$ as shown in Figure 7 . Thus, the length of connection duration is

$$
\tau=\frac{2 \sqrt{R^{2}-p_{y}^{2}}}{\left|v_{i}-v_{j}\right|} .
$$

With $s=2 \sqrt{R^{2}-p_{y}^{2}}$, we have the PDF for connection duration length as:

$$
f_{D C(\tau)}=\frac{s}{\tau^{2}} \frac{f_{v}\left(\frac{s}{\tau}\right)+f_{v}\left(-\frac{s}{\tau}\right)}{Q\left(\frac{v_{\min }-\mu}{\sigma}\right)-Q\left(\frac{v_{\max }-\mu}{\sigma}\right)}, \tau \geq 0 .
$$

The PDF of the connected duration length for vehicles on neighboring lanes with same and opposite moving direction are plotted in Figure 8. Compared with Figure 8(b), 


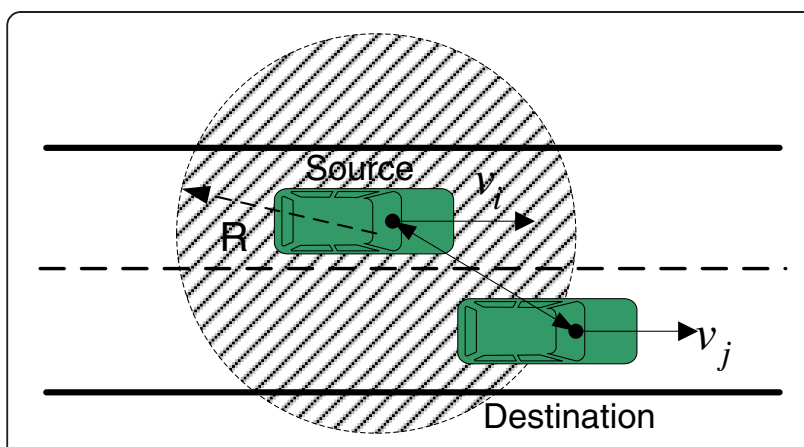

Figure 7 Vehicles on neighboring lanes.

it can be noticed that there is a large opportunity for vehicles connecting for a long time when they drive in the same direction, as shown in Figure 8(a). However, for vehicles moving with opposite direction as shown in
Figure $8(\mathrm{~b})$, the short connection duration occupies large portion of the probability density. The reason is apparent because of the different velocity differences for vehicles driving in different directions. This result also shows that to design underlying routing protocol for vehicles moving on the opposite direction, where routes may be only valid for a very short interval, is quite difficult than on the same direction.

\section{Indirect connectivity}

In this section, we will discuss the contributions to the available connectivity from indirect communication ways, which are further classified as multi-hop forwarding in a platoon and store-carry forwarding when no any instantaneous direct connection is achievable.

Considering a section of a unidirectional highway $H$ with length $[0, \mathrm{~L}]$ between the sender and the receiver

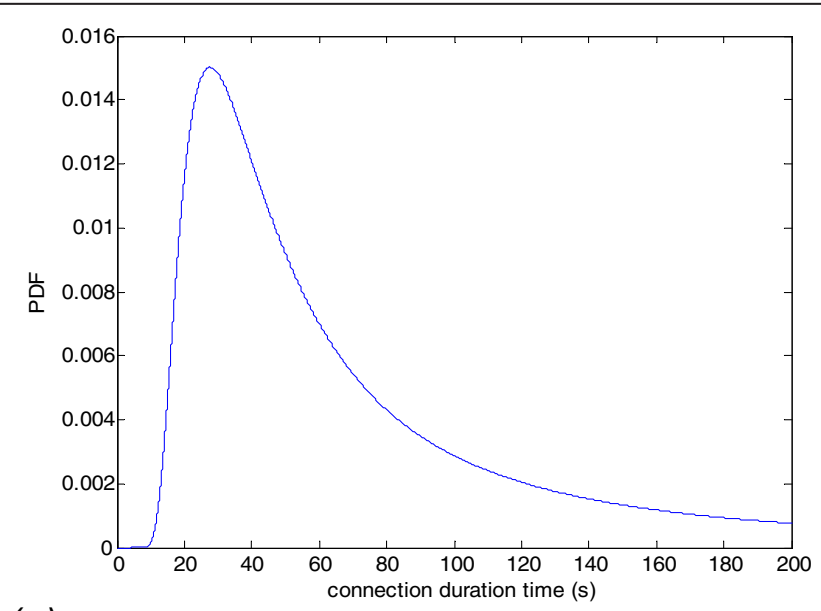

(a) PDF of connected duration for vehicles with same moving direction

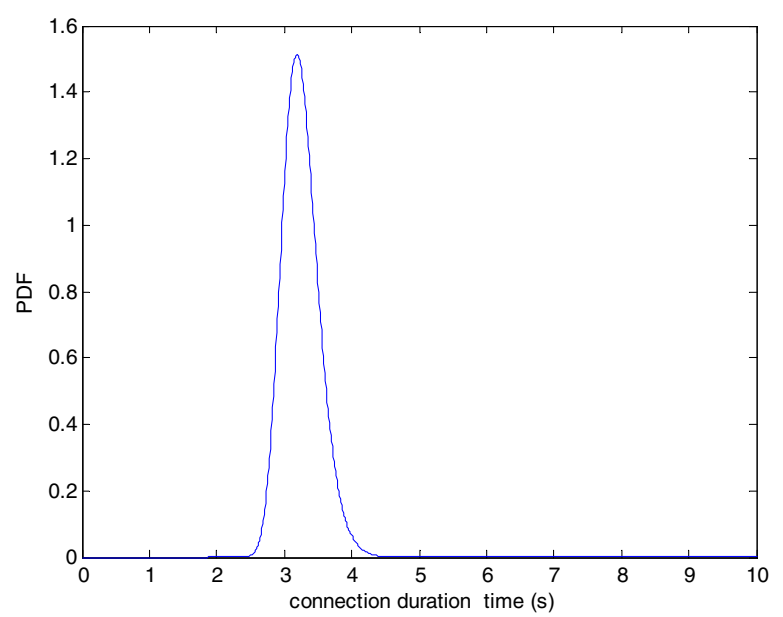

(b) PDF of connected duration for vehicles with opposite moving direction

Figure 8 PDF of connected duration for vehicles on neighboring lanes, with $\mu_{i}=\mu_{i}=30.8 \mathrm{~m} / \sigma, \sigma_{i}=\sigma_{j}=3.6 \mathrm{~m} / \mathrm{s}, r=100 \mathrm{~m}$. 
car, we will then give a more detailed analysis to the possible scenarios in which indirect connectivity can be obtained based on the above classifications.

\section{Indirect connectivity by multi-hop forwarding}

We will first investigate the achievable indirect connectivity based on multi-hop forwarding. Here, we discuss three cases: (1). Indirect connectivity in a platoon on a single lane when vehicles in this platoon are connected to each other; (2). Indirect connectivity for vehicles on a single lane with helps from vehicles in adjacent lanes, when multi-hop forwarding could not be obtained on this single lane; (3). Indirect connectivity for vehicles on multi-lane. In the first case, all vehicles in a lane are connected to each other forming a platoon. However, in the second case, there are communication gaps between vehicles in a single lane. Therefore, we introduce vehicles in adjacent lanes to fill the gaps and obtain the indirect connectivity. In the third case, by combining the above two cases, we obtained the general probability expression for multi-hop indirect connectivity in multi-lane scenario.

Case 1 Platoon is a typical mobility pattern of VANETs in which vehicles are forced to organize in platoons due to road congestion, legal speed limits, weather condition or other reasons resulting in the closing relative velocities between vehicles. A platoon can be defined as a set of autonomous vehicles which have to move in a convoy - i.e. following the path of the leader, through an intangible hooking [21]. Vehicles in the same direction are said to be within a platoon if and only if they can communicate with one another by one-hop or multi-hop way. Otherwise, vehicles are said to be in different platoons. To obtain the indirect connectivity in a platoon, the chained or multi-hop forwarding is an essential way by which packets are forwarded to the destination by relays. We then analyze the probability distribution of introduced connectivity by multi-hop forwarding in a platoon on a single lane.

Assuming that an observer stands at an arbitrary point of the highway without traffic lights, i.e., traffic is in the state of free flow state. It is widely accepted that the number of vehicles passing the observer per unit time follows a Poisson process [22], i.e. the arrival intervals are exponentially distributed. For simplicity, we assume that there are $M$ discrete levels of constant speed $v_{i}$, $i=1, \ldots, M$, on the highway, where the speeds are I.I.D for all vehicles and independent to their arrival intervals as we have stated before. Defining the communication radius as $r$, the arrival rate for vehicles with speed $v_{i}$ as $\lambda_{i}$, where $\sum_{i=1}^{M} \lambda_{i}=\lambda$, and then the occurrence probability for $v_{i}$ is $p_{i}=\frac{\lambda_{i}}{\lambda}$. Figure 9 illustrates the spacing distribution of vehicles with different speeds in a single lane. From [10], we can know that the inter-vehicle distances under such case are also I.I.D and exponentially distributed as follows:

$$
\begin{aligned}
p(L>x) & =1-F_{L}(x)=e^{-\sum_{i=1}^{M} \frac{\lambda_{i}}{v_{i}} x} \\
& =e^{-\lambda \sum_{i=1}^{M} \frac{p_{i}}{v_{i}} x}
\end{aligned}
$$

where $F_{L}(x)$ is the corresponding PDF of inter-vehicle distances.

Theorem 4: The Indirect connectivity on a single lane when vehicles in a platoon with $M$ constant speed levels are connected to each other is:

$$
\begin{aligned}
& p_{1}(\text { connectvity) } \\
& =\int_{v_{\min }}^{v_{\max }} \hat{f}_{\nu}(v)\left(e^{-\lambda_{i}^{\prime}}-\sum_{n=0}^{k} \frac{e^{-\lambda_{i}^{\prime}}}{n !} \gamma\left(n+1, r_{0} n \lambda^{\prime}{ }_{i}-\lambda_{i}^{\prime}{ }_{i}\right)\right) d v,
\end{aligned}
$$

where $\hat{f}_{v}(v)=\frac{2 f_{v}(\nu)}{\operatorname{erf}\left(\frac{v_{\max }-\mu}{\sigma \sqrt{2}}\right)-\operatorname{erf}\left(\frac{v_{\min }-\mu}{\sigma \sqrt{2}}\right)}$ and $f_{v}(v)=\frac{1}{\sqrt{2 \pi \sigma}} e^{-\frac{(v-\mu)^{2}}{2 \sigma^{2}}}$.

Proof From [23], for vehicles with speed $v_{i}$, the number of them within $\mathrm{H}$ follows a Poisson distribution with parameter $\lambda_{i}^{\prime}=\frac{L \lambda_{i}}{v_{i}}$. Then, the probability that these vehicles are connected is:

$$
p_{v_{i}}\left(r_{0}\right)=e^{-\lambda_{i}^{\prime}}-\sum_{n=0}^{k} \frac{e^{-\lambda^{\prime}}}{n !} \gamma\left(n+1, r_{0} n \lambda^{\prime}{ }_{i}-\lambda_{i}^{\prime}\right),
$$

for

$$
r_{0}=\frac{r}{L}, r_{0} \in L_{k}(\infty), k=1, \cdots, \infty
$$

Where

$$
\gamma(n, x)=\int_{0}^{x} t^{n-1} e^{-t} d t
$$

As shown in Figure 10, the platoon traffic with different speeds in a single lane can be equivalent to the superposition of traffics with a fixed speed in different lanes. Thereupon, the connected probability for a flow of vehicles with $M$ levels constant speed is:

$$
\begin{aligned}
p(\text { connectvity })= & \sum_{i} p_{i} p_{v_{i}}\left(r_{0}\right) \\
= & \sum_{i} p_{i}\left(e^{-\lambda_{i}^{\prime}}\right. \\
& \left.-\sum_{n=0}^{k} \frac{e^{-\lambda_{i}^{\prime}}}{n !} \gamma\left(n+1, r_{0} n \lambda_{i}^{\prime}-\lambda_{i}^{\prime}\right)\right)
\end{aligned}
$$

Based on the maximum and minimum speed limits in the highway, i.e. $v_{\max }$ and $v_{\min }$, by replacing $p_{i}, v_{i}$ in (16), 


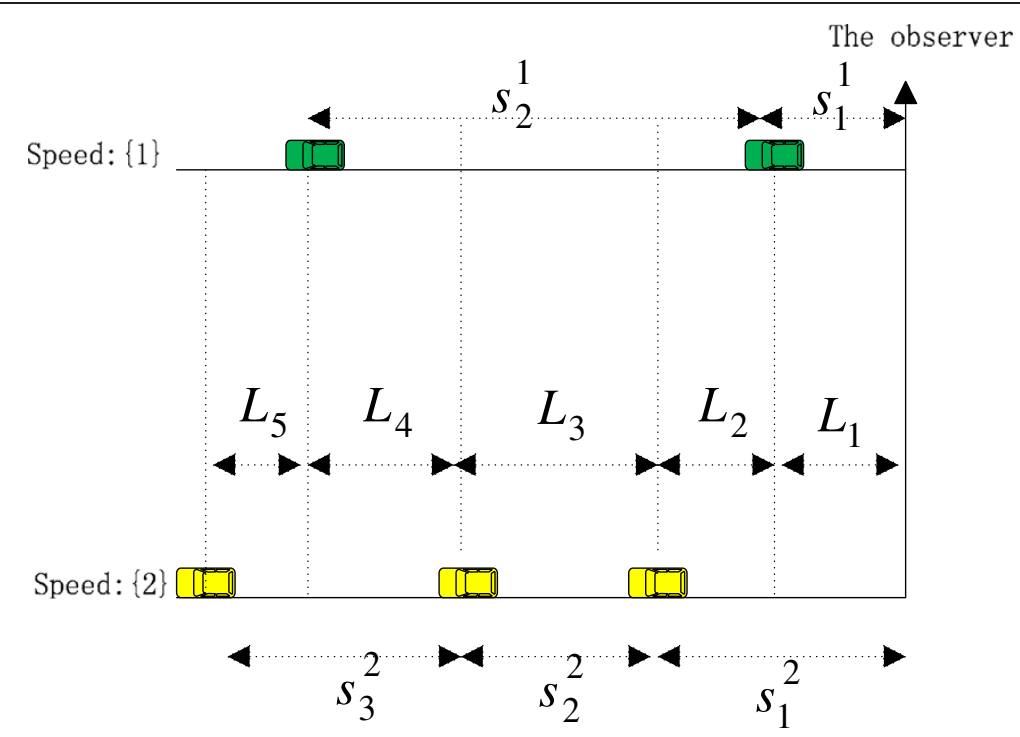

Figure 9 Example showing the distribution of the inter-vehicle distances with two speed levels. $s_{i}^{j}$ indicates the inter-vehicle distance between the vehicle $i-1$ and $i$ with the common speed level $j$, where $i=0$ indicates the position of the observer. $L_{i}$ indicates the inter-vehicle distance with different speeds.

We then get a truncated version of Equation (16) as (14) to avoid negative speed and zero speed.

Case 2 If the vehicles could not obtain connections on a single-lane, vehicles in adjacent lanes are needed to be introduced to provide direct links.

Assume $n$ independent nodes with radio range $r$ are uniformly distributed on $H$, i.e. $[0, \mathrm{~L}]$ of the highway. Then, from [24], the probability that a network is composed of at most $c$ platoons is:

$$
p_{c}=1-\sum_{i=c}^{m}(-1)^{i-c}\left(\begin{array}{c}
i-1 \\
c-1
\end{array}\right)\left(\begin{array}{c}
n-1 \\
i
\end{array}\right)\left(1-i \frac{r}{l}\right)^{n}
$$

where $m=\min \left\{n-1,\left\lfloor\frac{l}{r}\right\rfloor\right\}$.
Therefore, the average platoon number is:

$$
N_{\text {platoon }}=\sum_{c=1}^{\infty} p_{c} c
$$

Now, to explore the connection opportunities from adjacent lanes, we inspect the statistical characteristic between two platoons. We define the inter-platoon space as the distance between the last vehicle of the leading platoon and the first vehicle of the following platoon, and the intra-platoon space as the distance between the vehicles within the same platoon.

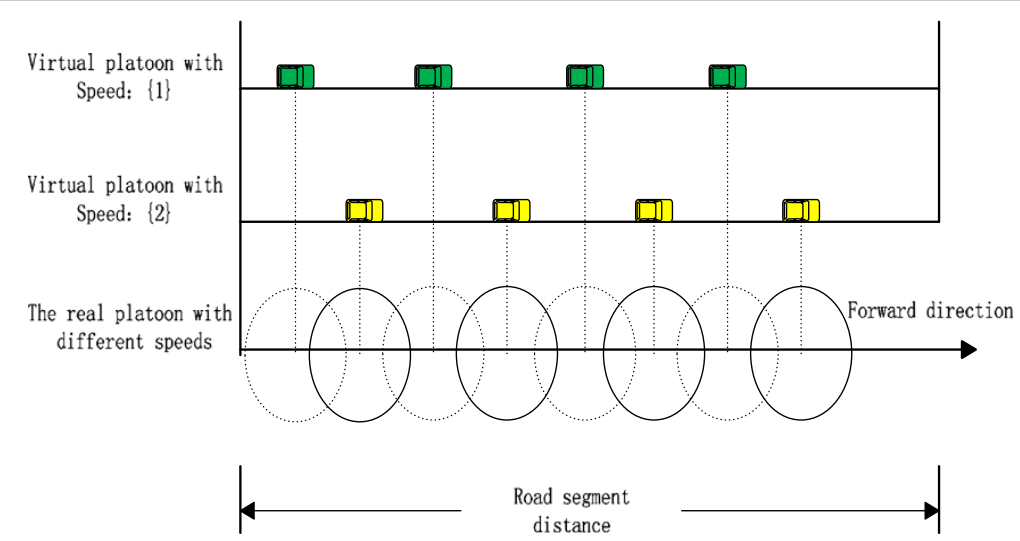

Figure 10 Simple example with two speed levels to show the computation procedure of the connectivity with different speed levels. 
Denoting inter-platoon space as $\mathrm{s}_{\text {inter }}$ the PDF of $\mathrm{s}_{\text {inter }}$ can be expressed as:

$$
\begin{aligned}
f_{s_{\text {inter }}}(s)= & p r[s \mid s>r] \\
= & \frac{\lambda \sum_{i=1}^{m} \frac{p_{i}}{v_{i}} e^{-\lambda \sum_{i=1}^{m} \frac{p_{i}}{v_{i}} s}}{e^{-\lambda \sum_{i=1}^{m} \frac{p_{i}}{v_{i}} r}} .
\end{aligned}
$$

Corollary 1: The expectation of $\mathrm{s}_{\text {inter }}$ is:

$$
E\left[s_{\text {inter }}\right]=\int_{r}^{\infty} s f_{s_{\text {inter }}}(s) d s=r+\frac{1}{\lambda \sum_{i=1}^{m} \frac{p_{i}}{v_{i}}} .
$$

Similarly, denoting intra-platoon space as $S_{\text {intra }}$, we can also get the PDF of $s_{\text {intra }}$ as:

$$
f_{s_{\text {intra }}}(s)=\operatorname{pr}[s \mid s<r]=\frac{\lambda \sum_{i=1}^{m} \frac{p_{i}}{v_{i}} e^{-\lambda \sum_{i=1}^{m} \frac{p_{i}}{v_{i}}}}{1-e^{-\lambda \sum_{i=1}^{m} \frac{p_{i}}{v_{i}}} .}
$$

Corollary 2: The corresponding expectation of $s_{\text {intra }}$ is:

$$
\begin{aligned}
E\left[s_{\text {intra }}\right] & =\int_{0}^{r} s f_{s_{\text {intra }}}(s) d s \\
& =\frac{1}{\lambda \sum_{i=1}^{m} \frac{p_{i}}{v_{i}}}-\frac{r e^{-\lambda \sum_{i=1}^{m} \frac{p_{i}}{v_{i}}}}{1-e^{-\lambda \sum_{i=1}^{m} \frac{p_{i}}{v_{i}}} .}
\end{aligned}
$$

Theorem 5: The probability that vehicles in a single-lane could be connected with helps from vehicles in adjacent lanes, when multi-hop forwarding could not be obtained on this single-lane is:

$$
P_{2}(\text { connectivity })=\left(\frac{e^{-\lambda E\left[s_{\text {inter }}\right]\left(\lambda E\left[s_{\text {inter }}\right]\right)^{\frac{E\left[s_{\text {inter }}\right]}{E\left[s_{\text {intra }}\right]}}}}{\left(\frac{E\left[s_{\text {inter }}\right]}{E\left[s_{\text {intra }}\right]}-1\right) !}\right)^{N_{\text {platoon }}-1}
$$

Proof To make platoons in a single-lane A connected, such as the following and the leading platoon in Figure 11, some vehicles paralleling to the communication gap between the two disconnected platoons in adjacent lanes are needed. With Equations (20) and (22), the average number of such vehicles can be expressed as:

$$
N=\frac{E\left[s_{\text {inter }}\right]}{E\left[s_{\text {intra }}\right]}-1 \text {. }
$$

Because the arrival processes of vehicles in adjacent lanes follow a Poisson distribution with density $\lambda E\left[s_{\text {inter }}\right]$, the probability that there need $N$ vehicles is:

$$
p(N)=\frac{e^{-\lambda E\left[s_{\text {inter }}\right]\left(\lambda E\left[s_{\text {inter }}\right]\right)^{N}}}{N !} .
$$

As a result, the probability that vehicles in a single lane could be connected with helps from vehicles in adjacent lanes is:

$$
P_{2}(\text { connectivity })=p(N)^{N_{\text {platoon }}-1} .
$$

With Equations (24), (25) and (26), (23) can be derived.

Case 3 Theorem 6: Based on the analysis of cases 1 and 2 , we can obtain the general expression for connectivity probability in multi-lane scenario as:

$$
\begin{aligned}
P(\text { connectivity })= & P_{1}(\text { connectivity }) \\
& +P_{2}(\text { connectivity }) .
\end{aligned}
$$

\section{Indirect connectivity by store-carry-forward}

In this subsection, we will analyze the indirect connectivity from store-carry-forward strategy where no any ongoing single-hop/multi-hop link is achievable between the sender and the intended receiver. We then discuss the store-carry-forward scenarios under the same-direction and opposite-direction cases respectively.

Same-direction store-carry-forward Figure 12 illustrates our discussed scene: when the sender breaks down, the forwarder will store its information and forward to the receiver. Obviously, this process contains two stages: stage 1, one forwarder receives the information from the sender; stage 2, one forwarder forwards the information to the receiver. Here, we define the vehicle on the front of potential forwarders as the "message head". In the first stage, if large speed difference exists among the possible forwarders, there may be possibility that the original "message head" is replaced by the subsequent fast one. Now, assuming these vehicles follow a Poisson arrival process with parameter $\lambda$ and their velocities are I.I.D and follow normal distribution, to explore the underlying store-carry-forward opportunities, we next analyze the movement feature of the message head.

Theorem 7: Assuming the message head at time $t$ is the vehicle with the maximum coordinate $x(t)$ among those vehicles passing the entrance during $(0, t]$. Thereupon, the CDF of its position at time $t$ is:

$$
F_{x(t)}=\sum_{n_{k}=0}^{\infty} p[M<x]^{n_{k}} \frac{e^{-\lambda t}(\lambda t)^{n_{k}}}{n_{k} !}
$$




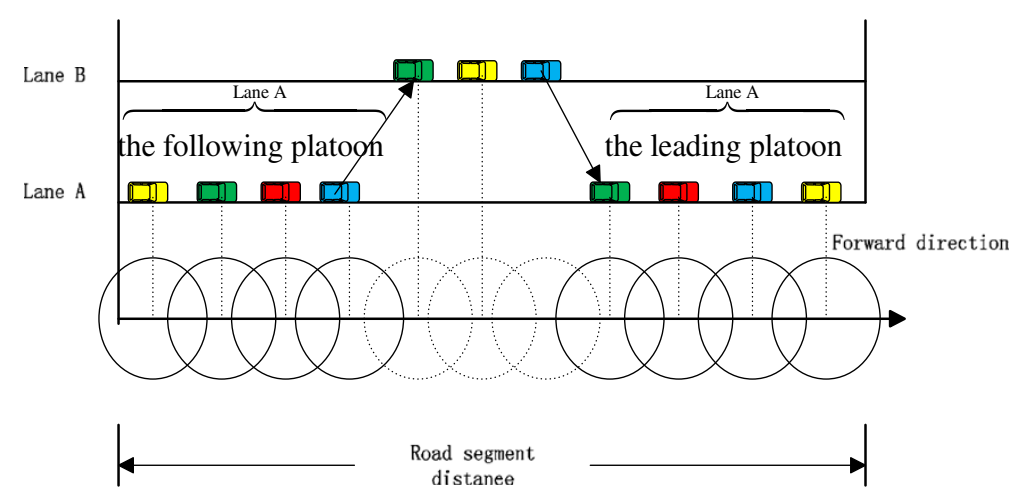

Figure 11 The scenario of relying on traffics from adjacent lanes to obtain indirect connectivity when full connectivity in a single lane could not be guaranteed.

where

$$
p(M<m)=\left\{\begin{array}{l}
\int_{u-3 \sigma}^{u+3 \sigma} \int_{0}^{\frac{m}{v}} m(v, z) d v d z, \frac{m}{t}<u-3 \sigma \\
\int_{\frac{m}{t}}^{u+3 \sigma} \int_{0}^{\frac{m}{v}} m(v, z) d v d z+ \\
\int_{u-3 \sigma}^{\frac{m}{t}} \int_{u+3 \sigma}^{t} m(v, z) d v d z, u-3 \sigma<\frac{m}{t}<u+3 \sigma \\
\int_{u-3 \sigma}^{t} \int_{0}^{t} m(v, z) d v d z, \frac{m}{t}>u+3 \sigma
\end{array},\right.
$$

and $m(v, z)=\frac{t-z}{t} \hat{f}_{v}(v)$.

Proof Define $N(t)$ as the number of vehicles passing the entrance during $(0, t]$. The "message head" at time $t$ is the vehicle with the maximum coordinate $x(t)$ among those vehicles passing the entrance during $(0, \mathrm{t}]$. Apparently, we can get:

$$
x(t)=\max \left(v_{i}\left(t-T_{i}\right)\right), i=1,2, \cdots, N(t),
$$

where $v_{i}$ is the average speed of vehicle $i$ and $T_{i}$ is the moment for vehicle $i$ passing the entrance. From [23], we know that values of $T_{i}$ are I.I.D and follow uniform $(0, t)$. Thereupon, the general PDF expressions of entering moment and velocity for these vehicles are $g(T)=\frac{T}{t}$ and $f(v)=\hat{f}_{v}(v)$. Then we can derive the $\operatorname{CDF}$ of $x(t)$ as:

$$
\begin{aligned}
& F_{x(t)}=\sum_{n_{k}=0}^{\infty} p\left[x(t)<x \mid N(t)=n_{k}\right] p\left[N(t)=n_{k}\right] \\
& =\sum_{n_{k}=0}^{\infty} p\left[v_{i}\left(t-T_{i}\right)<x, i=1,2, \cdots n_{k}\right] p\left[N(t)=n_{k}\right] \\
& =\sum_{n_{k}=0}^{\infty} p[v(t-T)<x]^{n_{k}} \frac{e^{-\lambda t}(\lambda t)^{n_{k}}}{n_{k} !}
\end{aligned}
$$

Denoting $Z=t-T$, then,

$$
\begin{aligned}
& p(Z<z)=p(t-T<z) \\
& =p(T>t-z) \\
& =1-p(T<t-z) \\
& =1-\int_{0}^{t-z} g(T) d T,
\end{aligned}
$$

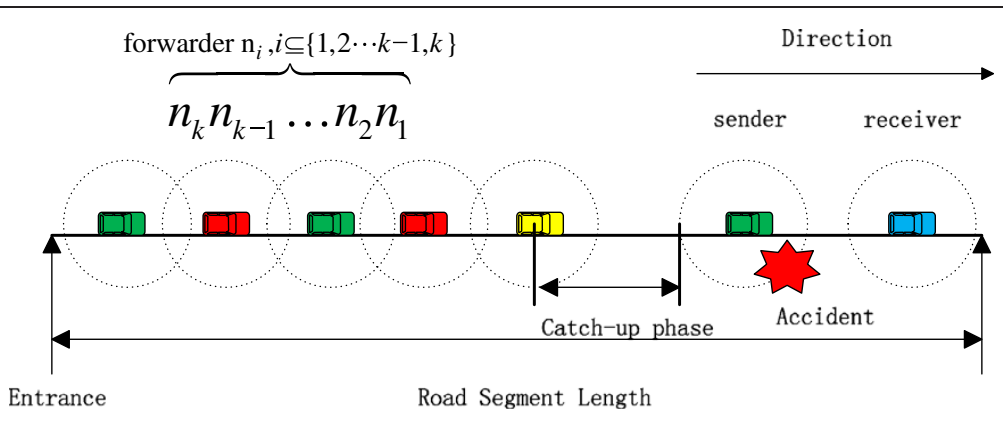

Figure 12 An example showing a break-down sender disseminates emergent notification depending on the forwarder. 
and the corresponding PDF is

$$
\begin{aligned}
& h(z)=p^{\prime}(Z<z) \\
& =g(t-z) . \\
& =\frac{t-z}{t}
\end{aligned}
$$

With $M=V Z$, the PDF of $M$ is

$$
\mathbf{\square}\left(m(v, z)=f(v) h(z) @=(t-, z) / t f \perp_{\downarrow}^{\dagger} v(v),\right)
$$

and the corresponding $\mathrm{CDF}$ is

$$
\begin{aligned}
& p(M<m)=p(V Z<m) \\
& =\left\{\begin{array}{l}
\int_{u-3 \sigma}^{u+3 \sigma} \int_{0}^{\frac{m}{v}} m(v, z) d v d z, \frac{m}{t}<u-3 \sigma \\
\int_{\frac{m}{t}}^{u+3 \sigma} \int_{0}^{\frac{m}{v}} m(v, z) d v d z+ \\
\int_{u-3 \sigma}^{\frac{m}{t}} \int_{u}^{t} m(v, z) d v d z, u-3 \sigma<\frac{m}{t}<u+3 \sigma \\
\int_{u-3 \sigma}^{t} \int_{0}^{t} m(v, z) d v d z, \frac{m}{t}>u+3 \sigma
\end{array} .\right.
\end{aligned}
$$

With Equations (30), (31), (32), (33) and (34), (28) can be derived.

Further, we can get the average velocity of the message head as:

$$
E[v(t)]=\frac{\partial[E[x(t)]]}{\partial t} .
$$

In the first stage, we can take all the forwarders as a virtual vehicle as shown in Figure 13. Therefore, the virtual vehicle has the coordinate distribution function $x(t)$ and the corresponding average velocity $E[v(t)]$.

Define $x_{\text {senden }} x_{\text {receiver }}$ as the location of the sender and the receiver respectively at time 0 . During the first stage, when the sender breaks down, the needed duration $\tau_{1}$ for messages disseminating from the sender to the forwarder at catch-up phase can be obtained by the following equation:

$$
x_{\text {sender }}-x\left(\tau_{1}\right)=r \text {. }
$$

In the second stage, the virtual vehicle keeps driving and forwards the message to the receiver when it moves into its radio range as shown in Figure 14.

Define $v_{\text {receiver }}$ as the speed of the receiver at location $x_{\text {receiver }}$ at time 0 . For the second stage, the needed duration $\tau_{2}$ for messages transmitting from the virtual vehicle to the receiver can be obtained from:

$$
x\left(\tau_{2}\right)+r=x_{\text {receiver }}+v_{\text {receiver }} \tau_{2} .
$$

Now, let us check the connection opportunities for the same-direction store-carry-forward case. Here, we just consider three vehicles, $\mathrm{A}, \mathrm{B}$ and $\mathrm{C}$ for simplicity, where $\mathrm{A}$ and $\mathrm{B}$ have the same velocity $v_{1}$ but $\mathrm{C}$ has velocity $v_{2}$, as shown in Figure 15. A, B and $\mathrm{C}$ are all out of radio range of each other. When A encounters an emergency such as an accident, it wants to disseminate the warning to others involving $\mathrm{B}$. However, because the distance $D 2=L$ between $\mathrm{A}$ and $\mathrm{B}$ is larger than radio range $r$ and both vehicles have the same speed, they cannot exchange information each other. Here, a forwarder is necessary to store the needed message from A and carry it to B by moving with a velocity $v_{2}$, where $v_{2}>v_{1}$. There are apparently two steps for this store-carry-forward process, i.e., the first step for packets exchange between $\mathrm{C}$ and $\mathrm{A}$ and the second step for packets exchange between $\mathrm{C}$ and $\mathrm{B}$. Before analyzing the connectivity possibility of this scenario, we first give some assumptions. As we have indicated, we just consider a unidirectional highway in this scene and there are only homogeneous traffic flows existent characterized by the lane-averaged velocities $v_{1}$ and $v_{2}$ and the overall vehicles density $\rho$. Furthermore, we assume a global market penetration ratio $a$ such that the working vehicle density just involves the equipped vehicles, i.e. $\vartheta=a \rho$. Meanwhile, for convenience, we place the sender A in a one-dimension Cartesian Coordinate system with fixed coordinate 0 , as shown in Figure 15.

Next, we investigate the statistical properties of the time $\tau_{1}$ needed for the first transversal hop from $\mathrm{C}$ to $\mathrm{A}$, where the distance between $\mathrm{C}$ and $\mathrm{A}$ is $D 1>r$.

We first introduce a stochastic variable $x$ defined by the PDF $f(x)=\vartheta e^{9 x} \theta(-x)$ expressing vehicle C's coordinate. The $\theta(x)$ is a function with result 1 when xis larger than zero and zero for others.

An instantaneous transversal hop for the generated message from $\mathrm{A}$ to the $\mathrm{C}$ is possible if $\mathrm{C}$ is within A's radio range, i.e. $-r \leq x \leq 0$, where $x$ is the position of $\mathrm{C}$. Then, the happening probability for this instantaneous transversal hop is

$$
p_{1}(0)=\int_{-r}^{0} f(x) d x=1-e^{-9 r} .
$$

If $x<-r$, a finite interval $\tau_{1}$ is needed for $\mathrm{C}$ moving into the radio range of $\mathrm{A}$.

Theorem 8: The CDF of $\tau_{1}$ could be given as:

$$
p_{1}\left(\tau_{1}\right)=1-e^{-9\left(v_{2}-v_{1}\right) \tau_{1}-9 r} .
$$




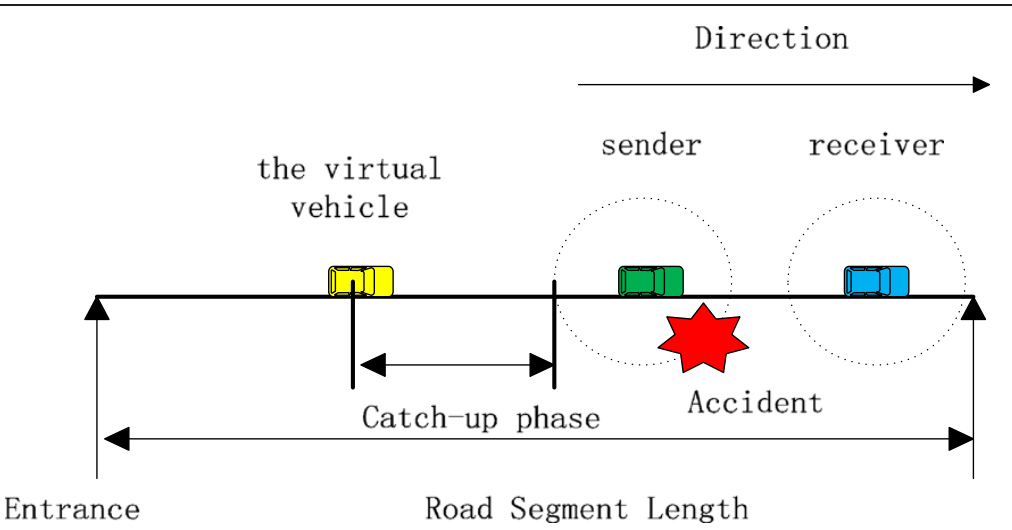

Figure 13 The first stage: the virtual vehicle receives the message sent by the sender. The virtual vehicle is equivalent to the forwarders in Figure 12 .

Proof Based on the relative velocity, i.e. $v_{2}-v_{1}$ between $\mathrm{C}$ and $\mathrm{A}$, the needed forwarding duration for $\mathrm{C}$ moving into the radio range of A could be calculated as $\tau_{1}=(-x-r) /\left(v_{2}-v_{1}\right)$. Therefore, a successful first transversal hop is possible at time $\tau_{1}$ or before it if the initial position of $\mathrm{C}$ meets $x \in\left(-\left(v_{2}-v_{1}\right) \tau_{1}-r, 0\right)$. As a result, the CDF of $\tau_{1}$ could be given as

$$
\begin{aligned}
p_{1}\left(\tau_{1}\right) & =\int_{-\left(\nu_{2}-v_{1}\right) \tau_{1}-r}^{0} f(x) d x \\
& =1-e^{-\vartheta\left(v_{2}-v_{1}\right) \tau_{1}-\vartheta r} .
\end{aligned}
$$

This CDF is plotted in Figure 16 with different $a$. It shows that given an overall vehicles density $\rho$ and velocities, the resulted $\tau_{1}$ is mainly influenced by the market penetration ratio $a$ and a larger $a$ could further reduce the needed duration for first transversal hop during the store-carry-forward procedure.

Then, we investigate the CDF of interval $\tau_{2}$ by which $\mathrm{C}$ could carry and forward the messages to $\mathrm{B}$.
Theorem 9: The CDF of $\tau_{2}$ could be expressed as:

$$
p_{2}\left(\tau_{2}\right)=1-e^{9\left[-\left(v_{2}-v_{1}\right) \tau_{2}+L-r\right]} .
$$

Proof $\tau_{2}$ could be derived from the expression of C's position at the moment of the second transversal hop when $\mathrm{C}$ moves into the radio range of $\mathrm{B}$, i.e. $x\left(\tau_{2}\right)=x+$ $\left(v_{2}-v_{1}\right) \tau_{2}$. Then the CDF of $\tau_{2}$ could be expressed as:

$$
\begin{aligned}
& p_{2}\left(\tau_{2}\right)=p\left(x\left(\tau_{2}\right)>L-r\right) \\
& =p\left(x>-\left(v_{2}-v_{1}\right) \tau_{2}+L-r\right) \\
& =\int_{-\left(v_{2}-v_{1}\right) \tau_{2}+L-r}^{0} f(x) d x . \\
& =\int_{-\left(v_{2}-v_{1}\right) \tau_{2}+L-r}^{0} \vartheta e^{9 x} \theta(-x) d x \\
& =1-e^{9\left[-\left(v_{2}-v_{1}\right) \tau_{2}+L-r\right]}
\end{aligned}
$$

The CDF of $\tau_{2}$ under different market penetration ratio is plotted in Figure 17 and the results are similar with Figure 16. Finally, with statistical analysis to $\tau_{1}$ and

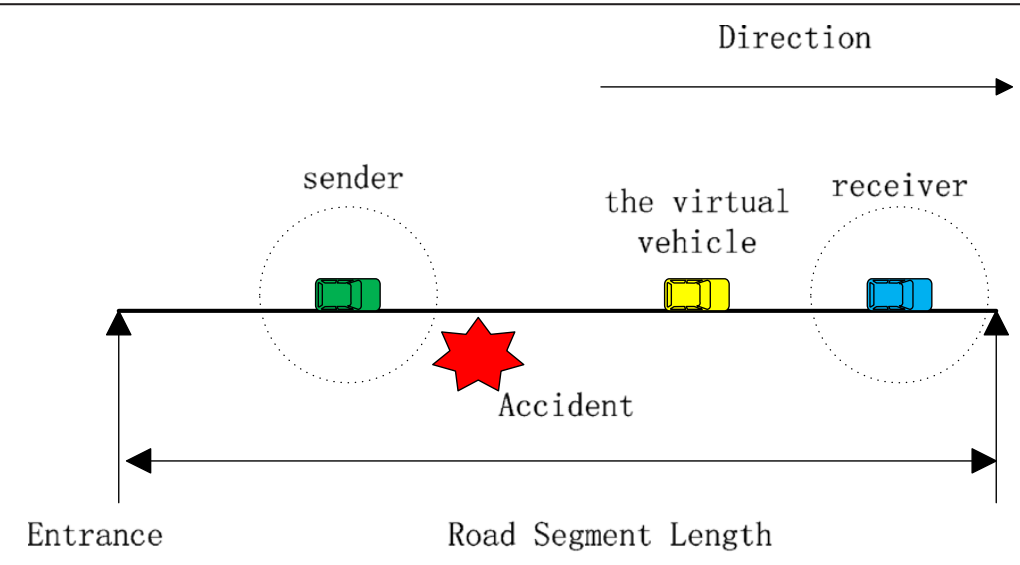

Figure 14 The second stage: the virtual vehicle sends messages received from the sender to the receiver. 


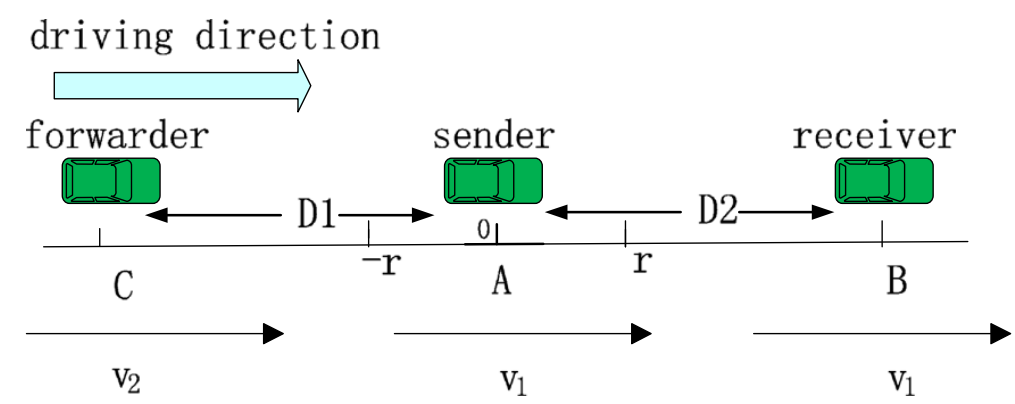

Figure 15 An example of store-carry-forward process.

$\tau_{2}$, the connection possibility from store-carry-forward when vehicles drive with the same direction could be obtained.

Opposite-direction store-carry-forward For store-carryforward cases where forwarders drive to the opposite direction, a better condition is that, by chance, there are forwarders within the radio range of the sender as shown in Figure 18(a). Instead, as shown in Figure 18(b), a worse case may also be possible when there is no any available vehicle as the store-carry forwarder within the radio range of the sender. In the worse case, the duration length for successful messages reception by the receiver is dependent on the time by which the available forwarder can occur. Therefore, the connection possibility under this worse case is stochastic and difficult to explore. Accordingly, we will just take the better case into consideration and analyze the corresponding statistical characteristics of connection duration.

We still assume that the distance between the sender and the receiver is $L$. The sender and the receiver have a

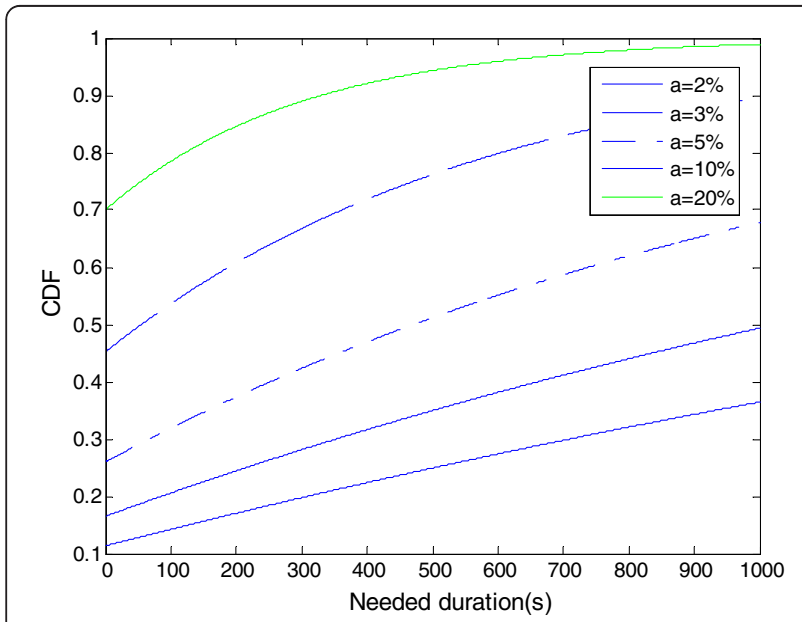

Figure 16 The CDF of $\tau_{1}$ under different market penetration ratio $a$ with $r=200 \mathrm{~m}, v_{1}=7 \mathrm{~km} / \mathrm{h}, v_{2}=9 \mathrm{~km} / \mathrm{h}, p=30 / \mathrm{km}$, $L=500 \mathrm{~m}$. constant speed $v_{1}$ whereas the store-carry forwarders drive in the opposite direction with $\operatorname{speed} v_{2}$. The radio ranges for all vehicles are $r$ and the forwarders are further assumed to form a connected platoon denoted by $Z$ as shown in Figure 18(a). Such hypothesis is reasonable because it equals to the case that there is only single available forwarder within radio range of the sender. In other words, regarding the little contribution to the final connection duration, we neglect the information propagation time among vehicles in $Z$.

Taking the sender as the reference point $r p$, the number of vehicles in $Z$ passing $r p$ follows a Poisson distribution with arrival rate $\lambda$. We use $\tau_{1}, \tau_{2}, \ldots, \tau_{n}$ to express the arrival time of each vehicle. Making $T_{i}=\tau_{i}-\tau_{i-1}, i=1$, $2, \ldots, n, \tau_{0}=0$, we assign $\left\{T_{i}, i=1,2, \ldots, n\right\}$ as the arrival intervals sequence for the forwarders. Here, $T i$ are I.I.D and follows a exponential distribution with parameter $\lambda$, namely the PDF is $f(\tau)=\lambda e^{-\lambda \tau}$. Because the inter-vehicle distance is less than $r$ in $Z$, the inter-vehicle interval is then less than $\frac{r}{v_{2}}$. In such case, the corresponding PDF of

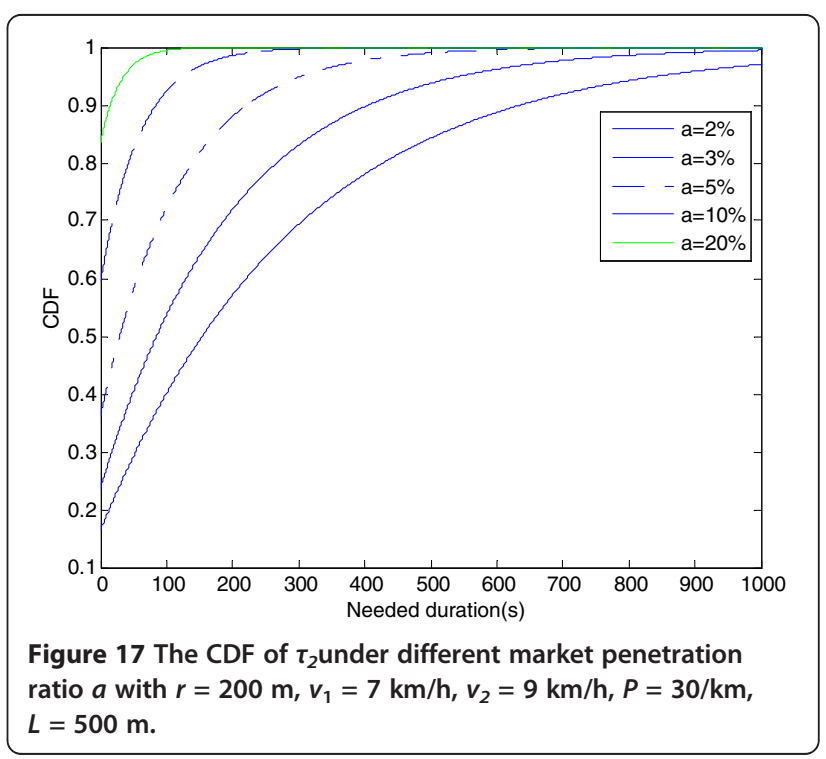




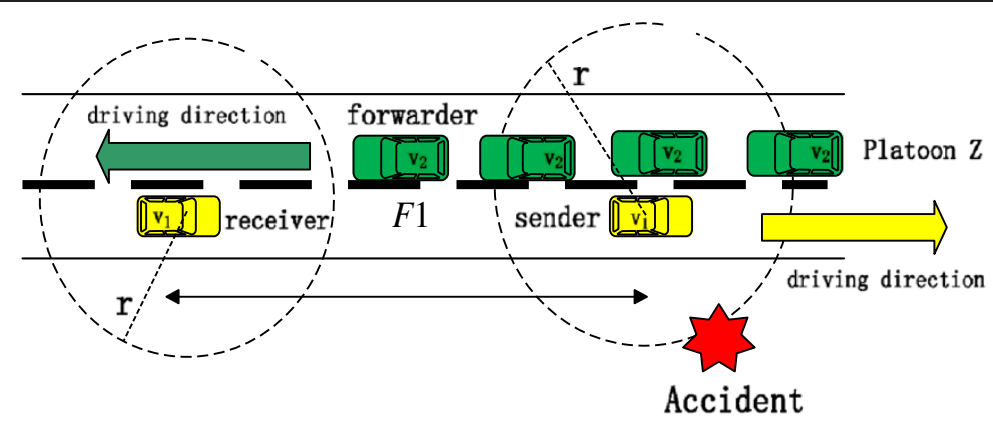

(a) Better case

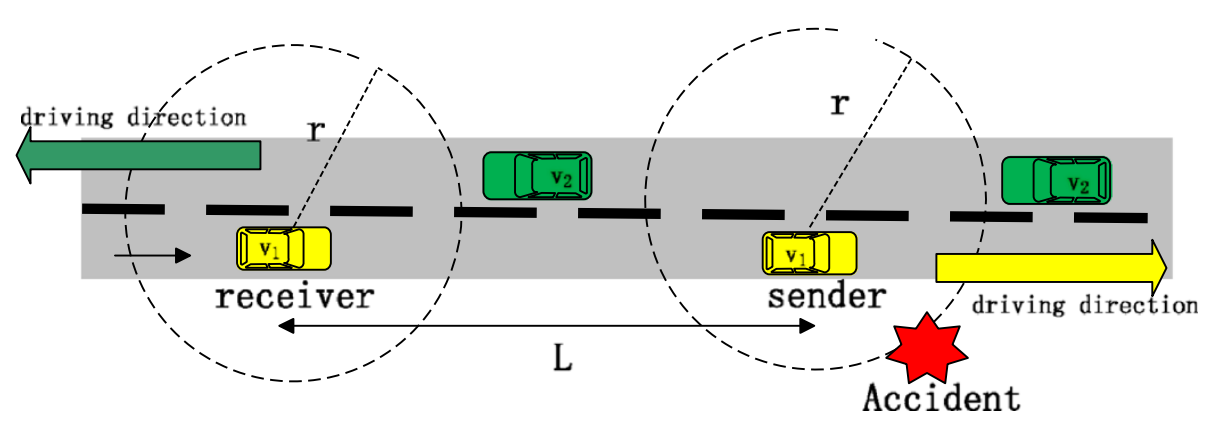

(b) Worse case

Figure 18 Store-carry-forward cases for vehicles driving in opposite direction.

inter-vehicle interval is:

$$
\begin{aligned}
f\left(\tau \mid \tau<\frac{r}{\nu_{2}}\right) & =\frac{f(\tau)}{p\left(\tau<\frac{r}{\nu_{2}}\right)} \\
& =\frac{f(\tau)}{\frac{r}{v_{2}} f(\tau) d \tau}=\frac{\lambda e^{-\lambda \tau}}{1-e^{-\lambda \frac{r}{v_{2}}}}
\end{aligned}
$$

Without loss of generality, we place the sender paralleling to the center of platoon $Z$. To analyze the needed duration for messages disseminating from the sender to the receiver by store-carry-forward, we consider the following two cases:

1. If there is any vehicle in $Z$ falling on the radio range of the receiver, with the above assumptions, we could take the needed duration ast ${ }_{1}=0$.

2. If there is no any vehicle in $Z$ falling on the radio range of the receiver, we should analyze CDF of the needed duration $t_{2}$ to further explore the connectivity characteristic.
Theorem 10: Assuming the number of vehicles between the sender and the receiver in platoon $Z$ is $N_{p}$, the sum of the arrival intervals between $N_{p}$ vehicles is $T$, where $T=\sum_{i=1}^{N_{p}} T_{i}$, then the PDF of $T$ is:

$$
f^{\left(N_{p}\right)}(T)=\frac{\lambda^{N_{p}} T^{\left(N_{p}-1\right)} e^{-\lambda T}}{\left(1-e^{-\lambda \frac{r}{v_{2}}}\right)^{N_{p}}\left(N_{p}-1\right) !},
$$

where $N_{p}=\left\lfloor\frac{e^{-\lambda \frac{r}{\nu_{2}}}}{2}\right\rfloor$.

Proof From [25], we know that the average number of vehicles, i.e. $C_{\text {platoon }}$ in a platoon is:

$$
E\left[C_{\text {platoon }}\right]=\frac{1}{P_{d}}
$$

where $P_{d}=1-\int_{0}^{\frac{r}{v_{2}}} f(\tau) d \tau=e^{-\lambda \frac{r}{v_{2}}}$.

Based on the assumption that the sender is paralleling to the center of $Z$, we have $N_{p}=\left\lfloor\frac{C_{p l a t o o n}}{2}\right\rfloor$. Therefore, the sum of the arrival intervals between the $N_{p}$ vehicles is 
$T=\sum_{i=1}^{N_{p}} T_{i}$. With $N_{p}$ fold convolution to the PDF of $T_{i}$, Equation (44) can be derived.

Corollary 3: The corresponding CDF of $T$ is

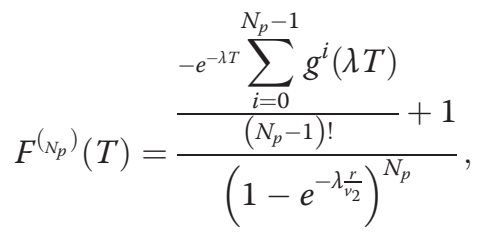

where $g(x)=x^{n}$ and $g^{n}(x)$ denote the $\mathrm{n}$ times differential to $g(x)$.

Theorem 11: The CDF of the needed duration $t_{2}$ is :

$$
\begin{aligned}
& p\left(t_{2}<t\right)=1-\frac{F^{\left(N_{p}\right)}\left(\frac{L-r-\left(v_{1}+v_{2}\right) t}{v_{2}}\right)}{F^{\left(N_{p}\right)}\left(\frac{L}{v_{2}}\right)}, \\
& L-r-\left(v_{1}+v_{2}\right) t>0 .
\end{aligned}
$$

Proof With the constant speed $v_{2}$, the sum of the total spaces between the $N_{p}$ vehicles is $l=\sum_{i=1}^{N_{p}} l_{i}$, where $l_{i}$ indicates the inter-vehicle distance and it could be also expressed as:

$$
l=v_{2} \cdot T
$$

Then the CDF of $l$ is

$$
\begin{aligned}
s(l) & =P\left(v_{2} \cdot T<l\right)=P\left(T<\frac{l}{v_{2}}\right) \\
& =F^{\left(N_{p}\right)}\left(\frac{l}{v_{2}}\right) .
\end{aligned}
$$

Therefore, the conditional CDF for $l<L$ is:

$$
s(l \mid l<L)=\frac{F^{\left(N_{p}\right)}\left(\frac{l}{v_{2}}\right)}{F^{\left(N_{p}\right)}\left(\frac{L}{v_{2}}\right)} .
$$

The needed duration for the first vehicle, marked as F1 in Figure 18(a) in platoon $Z$, entering into the radio range of the receiver is:

$$
t_{2}=\frac{L-r-l}{v_{1}+v_{2}}
$$

Therefore, the CDF of $t_{2}$ could be expressed as:

$$
\begin{aligned}
& p\left(t_{2}<t\right)=P\left(\frac{L-r-l}{v_{1}+v_{2}}<t\right) \\
& =1-P\left(l<L-r-\left(v_{1}+v_{2}\right) t\right) \\
& =1-s\left(L-r-\left(v_{1}+v_{2}\right) t l<L\right) \\
& =1-\frac{F^{(N)}\left(\frac{L-r-\left(v_{1}+v_{2}\right) t}{v_{2}}\right)}{F^{(N)}\left(\frac{L}{v_{2}}\right)}, \\
& L-r-\left(v_{1}+v_{2}\right) t>0 .
\end{aligned}
$$

Figure 19 shows the CDF of the needed duration $t_{2}$ in our discussed opposite store-carry-forward scenario. It can be noticed that the transmission radius greatly influence the required period for a successful store-carry-forward information exchange. Besides, there is a large probability for vehicles with larger transmission radius to successfully finish packets exchanging within short durations such as smaller than 8 seconds for $r=250 m$ as shown in Figure 19. However, there may need twice time for packets exchanging for vehicles just with $r=150 \mathrm{~m}$.

With aforementioned analysis, we could find that, in VANETs environment, the connectivity may be obtained by many different ways instead of only direct links. Based on the definition of available connectivity by Equation (2), the total achievable number of connections for a given topology at a determined moment could be calculated. This important statistic can be used to improve the network performance especially for safety-related applications where the vehicles with larger available connectivity may be the better candidates for emergent messages forwarding to make alerts spreading quickly and widely.

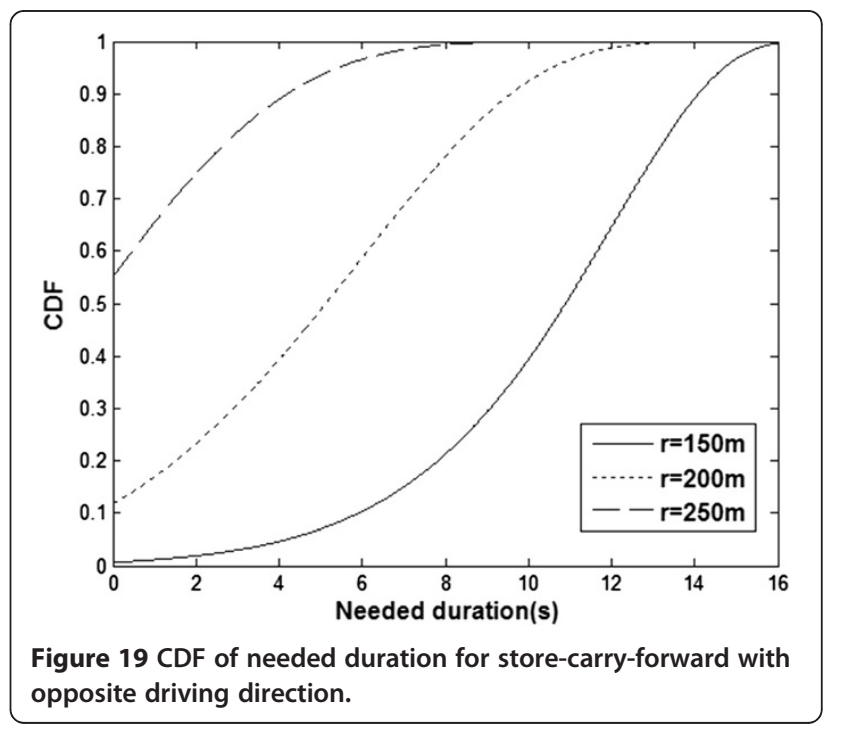




\section{Numerical results}

In this section, we will evaluate the performance of our proposed available connectivity measurement model in a predefined vehicular network. The simulation is executed on Matlab platform [26]. The setting for vehicles' speed is based on [27], which has given some referenced values listed in Table 2. Thirty-one vehicles are exponential distributed along a two-direction two-lane highway of length $10000 \mathrm{~m}$. The average value of this exponential distribution is $1000 /(\lambda \cdot A)$, where $\lambda$ is the vehicular arrival rate with unit vehicles/hour and $A$ is defined as $\int_{v_{\min }}^{v_{\max }} \frac{f_{V}(v)}{v} d v$ with unit hours $/ \mathrm{km} . r$ here indicates the radio radius with unit meters.

Figure 20 shows the $\mathrm{CDF}$ of available connectivity for the reference vehicle, i.e. vehicle (0), with different $\lambda, A$ and $r$. According to this figure, when the inter-vehicle space is smaller than $r$, the CDF is approximate to 1 . In other words, two equipped vehicles could connect to each other if they wish when the inter-vehicle distance is less than the radio range. However, when the intervehicle distance is larger than radio range $r$, CDF begins to decrease. Therefore, it can be concluded that the available connectivity is mainly determined by the radio range. But, the amplitude for CDF falling when Distance $>r$ is also determined by $\lambda$ and $A$. When $\lambda$ remains the same, a larger $A$ will increase the opportunity for connection due to its smaller average velocity and corresponding variance. For different values of $\lambda$, it is obvious that a bigger $\lambda$ will increase the equipped vehicle density on the investigated road segment with a fixed market penetration ratio. Thereupon, the available connection possibility will be enhanced.

Figure 21 shows the performance of our defined available connectivity metric for vehicle (0) with different $\lambda$, $A$ and $r$. The available connectivity is calculated per two seconds based on Equation (2), i.e. $\delta=2$. It is noted that the radio range $r$ is still the most influential factor on the results. With the same $\lambda$ and $A$, the available connectivity of $r=500 \mathrm{~m}$ has at most $67 \%$ improvement than the result of $r=350 \mathrm{~m}$. The influence of $\lambda$ to the results is a bit weaker than the radio range. When $A$ and $r$ keep the same, there is at most 32\% improvement of

\section{Table 2 Distribution parameters for vehicles' speed}

\begin{tabular}{lll}
\hline $\boldsymbol{\mu}[\mathbf{k m} / \mathbf{h}]$ & $\boldsymbol{\sigma}[\mathbf{k m} / \mathbf{h}]$ & $\mathbf{A}[\mathbf{h} / \mathbf{k m}]$ \\
\hline 70 & 21 & 0.016105 \\
90 & 27 & 0.012526 \\
110 & 33 & 0.010249 \\
130 & 39 & 0.008672 \\
150 & 45 & 0.007516 \\
\hline
\end{tabular}

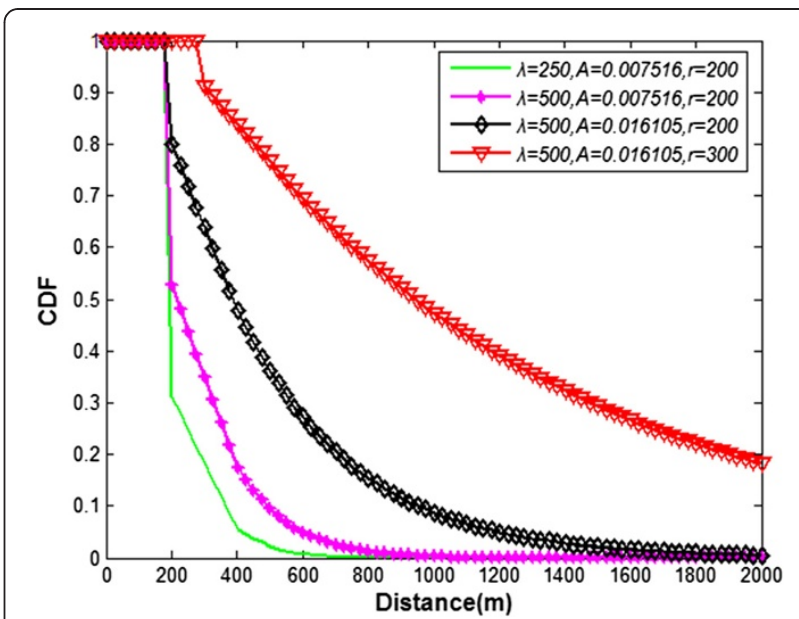

Figure $20 \mathrm{CDF}$ of available connectivity for vehicle (0) with different $\lambda, A, r$.

available connectivity for $\lambda=500$ to $\lambda=250$. The impact of $A$ on the result is the same as shown in Figure 20, say that a larger $A$ will result in a bigger available connectivity due to moderate topology changing.

The impact of velocity on our proposed available connectivity has been shown in Figure 22 with different normal distribution parameters $\mu, \sigma$. It can be noticed that the available connectivity is mainly influenced by the average velocity. A smaller average speed will bring more connection opportunities to vehicles and this conclusion is consistent with the results from Figures 20 and 21. For velocity variance, it is interested that a bigger value will result in a larger available connectivity. This phenomena also verify the results of Figures 20 and 21 where a larger $\sigma$ corresponds to a bigger $A$

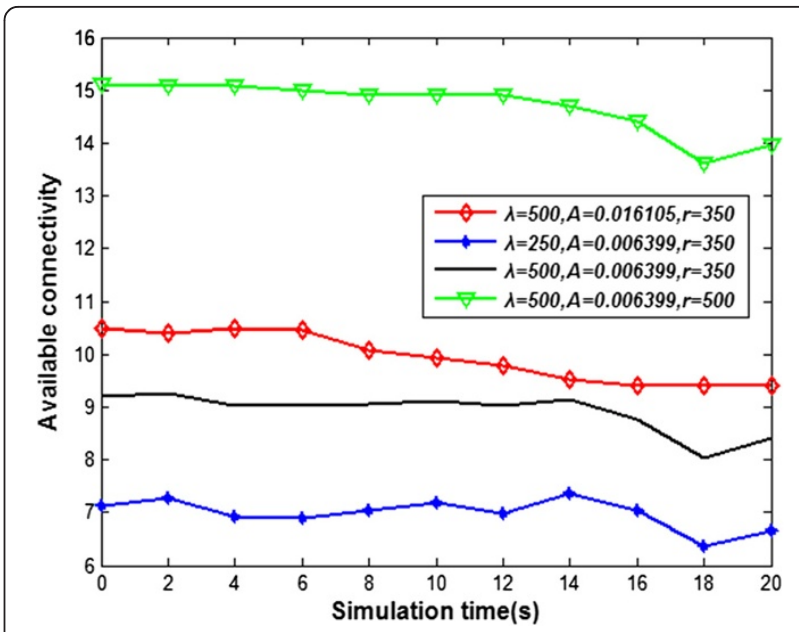

Figure 21 Available connectivity for vehicle (0) with different $\lambda$, $A, r$. 


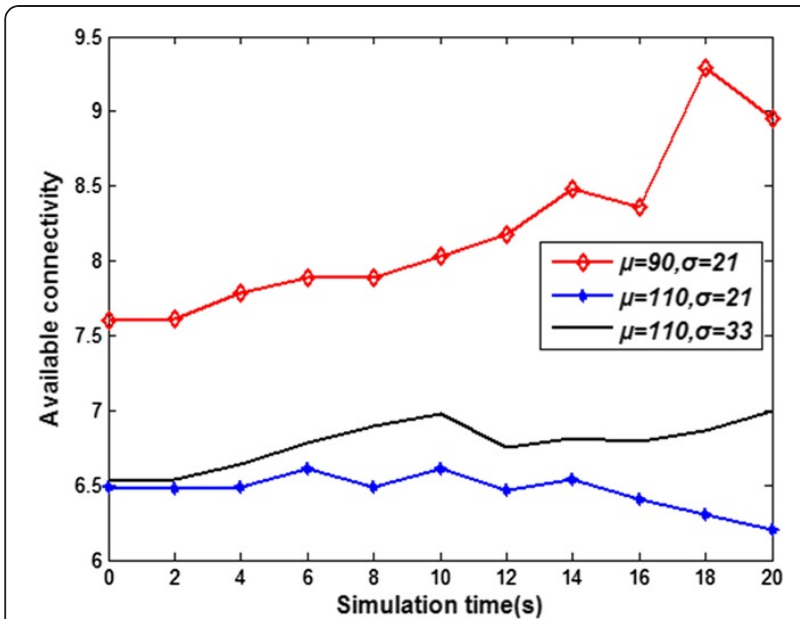

Figure 22 Available connectivity for vehicle (0) with different $\mu, \sigma$.

resulting in more available connectivity when $\mu$ remains the same.

To verify our numerical results with real data, we also implement the available connectivity measurement model on our test bed. Figure 23 depicts the simulation scenario with eight toy vehicles on two lanes. The vehicle has two operation modes, i.e. infrared controlling or automatic piloting. In automatic piloting pattern, the car can be programmed to a fixed speed with minimum limit $50 \mathrm{~cm} / \mathrm{s}$ and maximum limit $10 \mathrm{~m} / \mathrm{s}$ respectively. Thereupon, we can generate vehicles' speed according to normal distribution given later. To evaluate the connectivity performance, we launched a wireless communication device on each vehicle. This device consists of an ATMEL Atmega128L MCU (Microprogrammed Control Unit), a Chipcon 2420 SmartRF, a Maxim IC MAX3232 transceiver and some other chips for storage and debug. To code our model, we also implemented an embedded GNU Compiler Collection environment with a simplified operating system on this device.

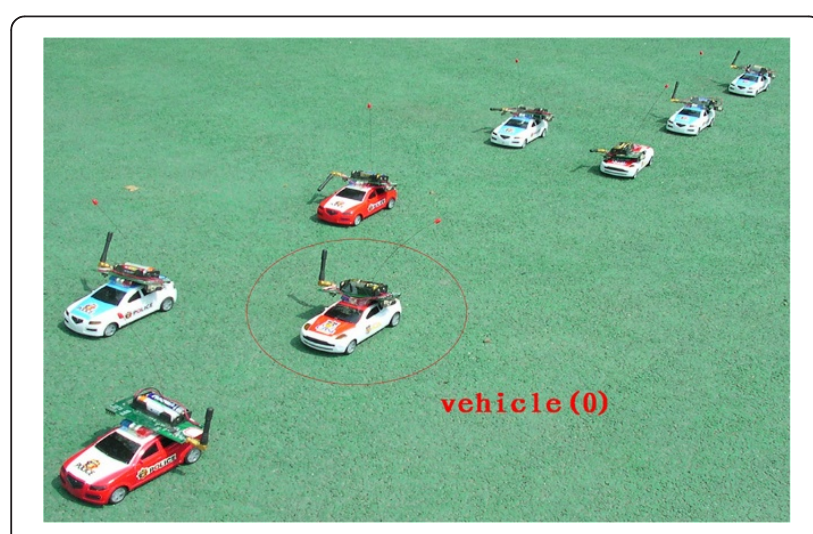

Figure 23 Testbed scenario for available connectivity measurement.
Table 3 Distribution parameters for toy vehicles' speed

\begin{tabular}{lll}
\hline $\boldsymbol{\mu}[\mathbf{k m} / \mathbf{h}]$ & $\boldsymbol{\sigma}[\mathbf{k m} / \mathbf{h}]$ & $\mathbf{A}[\mathbf{h} / \mathbf{k m}]$ \\
\hline 32.2 & 6.4 & 0.032407 \\
17.6 & 2.8 & 0.058327 \\
\hline
\end{tabular}

To make our simulation practical, we scale down the corresponding test parameters for highway on our testbed. Table 3 shows the speed setting for our toy vehicle. Its size is $20 \mathrm{~cm}$ (length) $\times 7.5 \mathrm{~cm}$ (width). The length of test field is $40 \mathrm{~m}$ long and the arrival process follows an exponential distribution with $\lambda=500$ vehicles/hour. Figure 24 shows the CDF of available connectivity for vehicle (0), which has been pointed out in the picture, with different $A$, $r$ on our testbed. The radio range is set to 8 or 12 meters. It can be noticed that there are the same conclusions as shown in Figure 20. The radio range is still the most influential factor. When $r$ remains the same, a larger $A$ will increase the connection opportunities. This real data results further verify our assumptions and theoretical analysis. It also confirms the feasibility of our model.

\section{Conclusion}

In this article, a useful metric, i.e. available connectivity has been introduced in VANETs. The available connectivity not only comes from direct connections from neighbors, but also from the indirection links by multi-hop and store-carry forwarding. The elaborate investigation to the statistical properties of the available connectivity has been given. Numerical results show that our proposed available connectivity has many potential relationships with network parameters and may provide important references for future protocols design in VANETs.

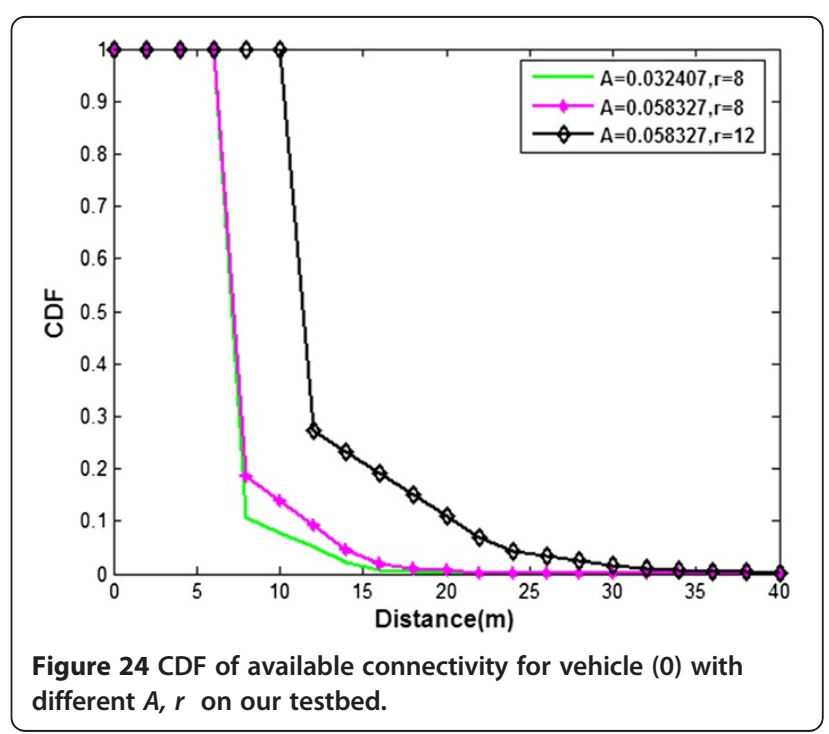




\section{Competing interests}

The authors declare that they have no competing interests.

\section{Acknowledgements}

This work was supported by the National Natural Science Foundation of China (61201133; 61172055;60832005; U0835004; 61072067), the Postdoctoral Science Foundation of China (20100481323), the Program for New Century Ex-cellent Talents (NCET-11-0691), the "111 Project" of China (B08038), and the Foundation of Guangxi Key Lab of Wireless Wideband Commu-nication \& Signal Processing (11105).

Received: 27 March 2012 Accepted: 17 July 2012

Published: 23 August 2012

\section{References}

1. L. Bononi, M. Di Felice, A cross layered mac and clustering scheme for efficient broadcast in vanets (Proc IEEE International Conference on Mobile AdHoc and Sensor Systems (MASS), Pisa, Italy, 2007), pp. 1-8

2. S. Eichler, Performance evaluation of the IEEE 802.11 p WAVE communication standard (Proc 66th IEEE Vehicular Technology Conference(VTC), Baltimore, MD, USA, 2007), pp. 2199-2203

3. T. El Batt, S.K. Goel, G. Holland, H. Krishnan, J. Parikh, Cooperative collision warning using dedicated short range wireless communications (Proc 3rd ACM international workshop on Vehicular ad hoc networks, New York, USA, 2006), pp. 1-9

4. A. Carter, The status of vehicle-to-vehicle communication as a means of improving crash prevention performance (Tech. Report, 05-0264, National Highway Traffic Safety Administration, USA, )

5. E. Schoch, F. Kargl, M. Weber, T. Leinmuller, Communication patterns in VANETs. IEEE Commun. Mag. 46(11), 119-125 (2008). doi:10.1109/MCOM.2008.4689254

6. H. Hartenstein, K.P. Laberteaux, A tutorial survey on vehicular ad hoc networks. IEEE Commun. Mag. 46(6), 164-171 (2008). doi:10.1109/MCOM.2008.4539481

7. M. Torrent-Moreno, M. Killat, H. Hartenstein, The challenges of robust intervehicle communications (Proc 62nd IEEE Vehicular Technology Conference (VTC), Dallas, USA, 2005), pp. 319-323

8. R. Nagel, The effect of vehicular distance distributions and mobility on VANET communications (Proc IEEE Intelligent Vehicles Symposium (IV), San Diego, CA, USA, 2010), pp. 1190-1194

9. A. Abul-Magd, Modeling highway-traffic headway distributions using superstatistics. Phys. Rev. E 76(5), 057101 (2007)

10. S. Yousefi, E. Altman, R. El-Azouzi, M. Fathy, Analytical model for connectivity in vehicular ad hoc networks. IEEE Trans. Veh. Technol. 57(6), 3341-3356 (2008). doi:10.1109/TVT.2008.2002957

11. C.P. Young, B.R. Chang, J.J. Lin, R.Y. Fang, Cooperative collision warning based highway vehicle accident reconstruction (Proc 8th International Conference on Intelligent Systems Design and Applications(ISDA), Kaohsiung, Taiwan, 2008), pp. 561-565

12. A. Cardote, S. Sargento, P. Steenkiste, On the connection availability between relay nodes in a VANET (Proc IEEE GLOBECOM Workshops (GC Wkshps), Miami, FL, USA, 2010), pp. 181-185

13. I.W.H. Ho, K.K. Leung, Node connectivity in vehicular ad hoc networks with structured mobility (Proc 32nd IEEE Conference on Local Computer Networks (LCN), Dublin, Ireland, 2007), pp. 635-642

14. M.M. Artimy, W. Robertson, W.J. Phillips, Connectivity in inter-vehicle ad hoc networks (Proc Canadian Conference on Electrical and Computer Engineering, Niagara Falls, Ontario, Canada, 2004), pp. 293-298

15. D.P. Spanos, R.M. Murray, Robust connectivity of networked vehicles (Proc 43rd IEEE Conference on Decision and Control(CDC), Atlantis, Paradise Island, Bahamas, 2004), pp. 2893-2898

16. M.M. Artimy, W.J. Phillips, W. Robertson, Connectivity with static transmission range in vehicular ad hoc networks (Proc 3rd IEEE Communication Networks and Services Research Conference, Halifax, Nova Scotia, Canada, 2005), pp. 237-242

17. M. Kafsi, P. Papadimitratos, O. Dousse, T. Alpcan, J.P. Hubaux, Vanet connectivity analysis. Arxiv preprint. arXiv 0912, 5527 (2009)

18. S. Durrani, Z. Xiangyun, A. Chandra, Effect of vehicle mobility on connectivity of vehicular ad hoc networks (Proc 72nd IEEE Vehicular Technology Conference Fall (VTC), Ottawa, Canada, 2010), pp. 1-5
19. M. Khabazian, M. Ali, A performance modeling of connectivity in vehicular networks. IEEE Trans. Veh. Technol. 57(4), 2440-2450 (2008). doi:10.1109/TVT.2007.912161

20. H. Wu, R.M. Fujimoto, G.F. Riley, M. Hunter, Spatial propagation of information in vehicular networks. IEEE Trans. Veh. Technol. 58(1), 420-431 (2009)

21. S. Colin, A. Lanoix, O. Kouchnarenko, J. Souquières, Using CSP\|B components: application to a platoon of vehicles. LNCS 5596, 103-118 (2009). doi:10.1007/978-3-642-03240-0_11

22. W. Feller, An Introduction to Probability Theory and Its Applications, vol. 2 (Wiley, India, 2008)

23. J. Wu, Connectivity analysis of a mobile vehicular ad hoc network with dynamic node population (Proc IEEE GLOBECOM Workshops(GC Wkshps), New Orleans, USA, 2008), pp. 1-8

24. A. Ghasemi, S. Nader-Esfahani, Exact probability of connectivity onedimensional ad hoc wireless networks. IEEE Commun. Lett. 10(4), 251-253 (2006). doi:10.1109/LCOMM.2006.1613737

25. N. Wisitpongphan, F. Bai, P. Mudalige, V. Sadekar, O. Tonguz, Routing in sparse vehicular ad hoc wireless networks. IEEE J. Sel. Areas Commun. 25(8), 1538-1556 (2007). doi:10.1109/JSAC.2007.071005

26. Matlab User's Guide (The MathWorks. Inc, Natick, MA, 1998), p. 5

27. M. Rudack, M. Meincke, M. Lott, On the dynamics of ad hoc networks for inter-vehicles communications (IVC) (Proc ICWN, Las Vegas, Nevada, USA, 2002), pp. 40-48

doi:10.1186/1687-1499-2012-270

Cite this article as: Chen et al:: Available connectivity analysis under free flow state in VANETs. EURASIP Journal on Wireless Communications and Networking 2012 2012:270.

\section{Submit your manuscript to a SpringerOpen ${ }^{\odot}$ journal and benefit from:}

- Convenient online submission

- Rigorous peer review

- Immediate publication on acceptance

- Open access: articles freely available online

- High visibility within the field

- Retaining the copyright to your article

Submit your next manuscript at springeropen.com 